University of Texas at El Paso

\title{
DigitalCommons@UTEP
}

Open Access Theses \& Dissertations

2017-01-01

\section{A Novel Approach to Enhancing Upper Extremity Coordination in Children with Autism Spectrum Disorder}

Alejandra Sarahi Gamez Corral

University of Texas at El Paso, asgamezco@gmail.com

Follow this and additional works at: https://digitalcommons.utep.edu/open_etd

Part of the Kinesiology Commons

\section{Recommended Citation}

Gamez Corral, Alejandra Sarahi, "A Novel Approach to Enhancing Upper Extremity Coordination in Children with Autism Spectrum Disorder" (2017). Open Access Theses \& Dissertations. 450.

https://digitalcommons.utep.edu/open_etd/450 


\title{
A NOVEL APPROACH TO ENHANCING UPPER EXTREMITY COORDINATION IN CHILDREN WITH AUTISM SPECTRUM DISORDER
}

\author{
ALEJANDRA SARAHI GAMEZ CORRAL \\ Master's Program in Kinesiology
}

APPROVED:

Jason B. Boyle, Ph.D., Chair

Harry Meeuwsen, Ph.D.

Rhonda Manning, PT, DPT, PCS.

Charles Ambler, Ph.D.

Dean of the Graduate School 
Copyright (C)

by

Alejandra S. Gamez Corral

2017 


\section{Dedication}

This work of many to come is dedicated to all the children diagnosed with Autism Spectrum Disorder and to my family who have always supported me in my endeavors. 
A NOVEL APPROACH TO ENHANCING UPPER EXTREMITY COORDINATION IN CHILDREN WITH AUTISM SPECTRUM DISORDER

by

ALEJANDRA SARAHI GAMEZ CORRAL

THESIS

Presented to the Faculty of the Graduate School of

The University of Texas at El Paso

in Partial Fulfillment

of the Requirements

for the Degree of

MASTER OF SCIENCE

Kinesiology Department

THE UNIVERSITY OF TEXAS AT EL PASO

December 2017 


\section{Acknowledgements}

This work would have not been possible without the help and constant support of Dr.

Jason B. Boyle, Dr. Rhonda Manning, and Dr. Harry Meeuwsen. Thank you for the wonderful experience of working with you and for allowing me to see the beauty behind research.

Thank you to The Organization for Autism Research (OAR) for honoring this project with the 2016 Graduate Research Grant. 


\begin{abstract}
With current estimates of 1 in 68 children being affected, Autism Spectrum Disorder (ASD) is regarded as one of the most rampant forms of disability globally. Although typically diagnosed through social impairment and repetitive stereotypical behavior, recent work has shown distinct motor impairments across the autism spectrum. These motor deficits can be gross or fine in nature and can be seen not only in reaching and grasping, but also in the kinematic composition of the actual movement, such as decreased velocity, decreased accuracy, irregular movement smoothness, etc. The goal of this study was to further the understanding of the kinematic components of goal directed upper limb movement in participants with ASD and to investigate the adaptability of their limb movements after performing a sine wave tracking task. A custom-built bi-manual arm bar apparatus was used, which allowed frictionless bimanual flexion and extension of the limbs in the elbow joint in the horizontal plane. Positional data of the limbs displacement was integrated into a custom built graphic user interface platform that relayed information regarding the position of the participant's limbs on a projection screen. The participants tracked a sine wave template and performed a speed-accuracy trade-off (Fitts) task that involved moving a cursor between two targets of varying sizes and separation distances. Data analysis examined kinematics before, during, and after both tasks. Variables of interest were: Movement time, peak velocity, \% time to peak velocity, slowing parameters, end point accuracy and harmonicity. The findings from this study indicate that children with ASD respond just as well to the tracking task as typical developing children by executing post-training movements of higher velocity with smoother displacement profiles.
\end{abstract}




\section{Table of Contents}

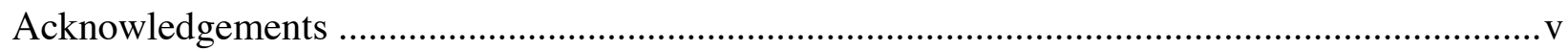

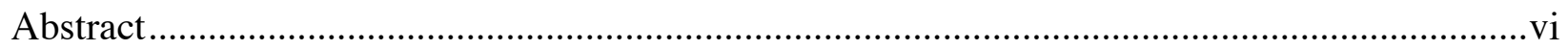

Table of Contents ................................................................................................................

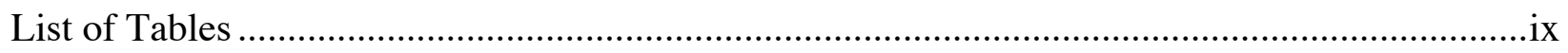

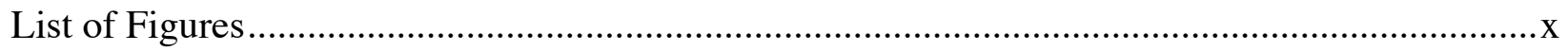

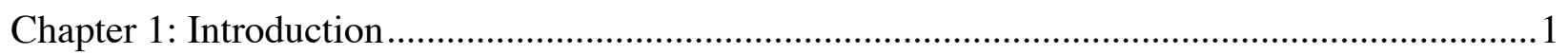

1.1 What is Autism Spectrum Disorder (ASD)? ………….........................................

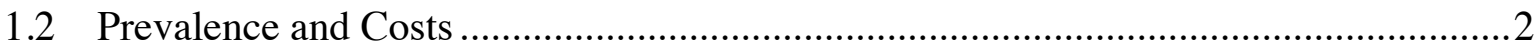

1.3 Motor Impairments in Children with ASD ..................................................................

1.3.1 Gross and Fine Motor Impairments ...........................................................

1.3.2 Motor Planning and Control Impairments .....................................................

1.3.3 Gestures and Motor Imitation Impairments ……………………...................5

1.3.4 Early Motor Impairments ........................................................................6

1.3.5 Cerebellum Dysfunction .........................................................................

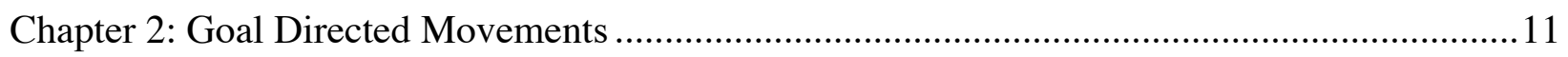

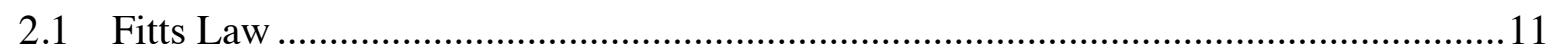

2.2 Novel Sine Wave Tracing Task ............................................................................

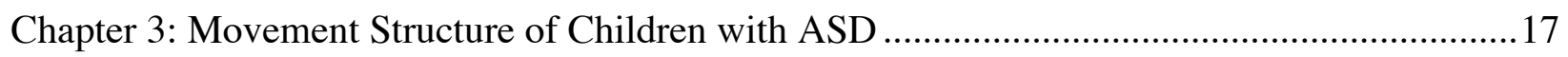

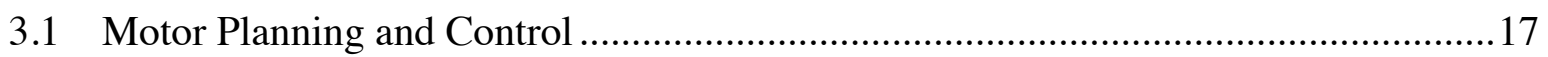

3.1.1 Motor Planning Impairments ....................................................................... 17

3.1.2 Motor Control Impairments ...................................................................20

3.2 Motor Planning and Control Analysis Utilizing Fitts (1954) Paradigm.......................23

Chapter 4: Novel Approach to Enhancing Coordination in Children with ASD ...........................25

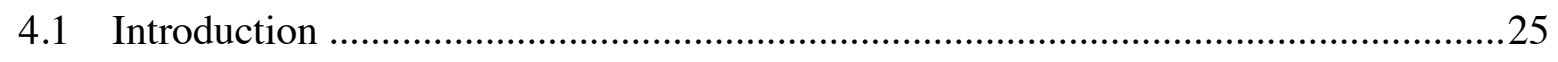

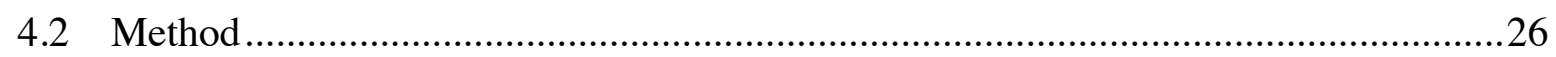

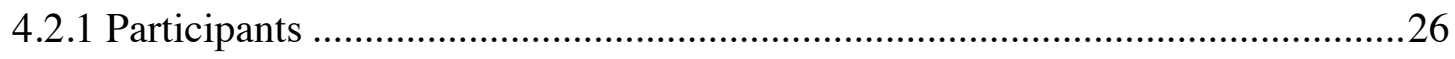

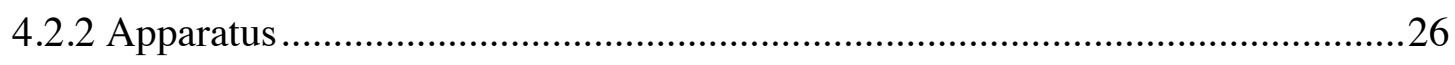




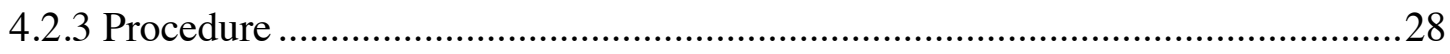

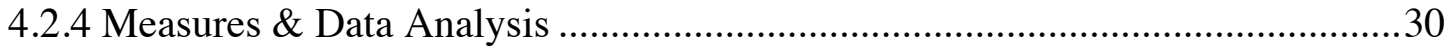

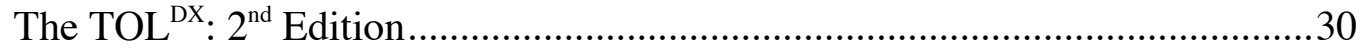

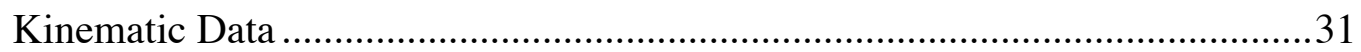

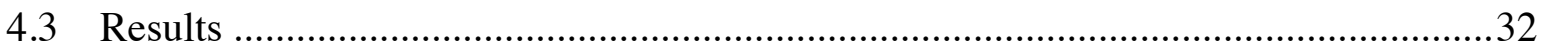

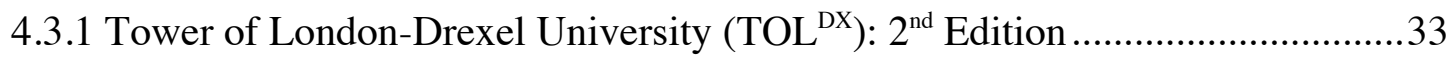

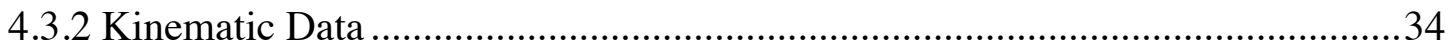

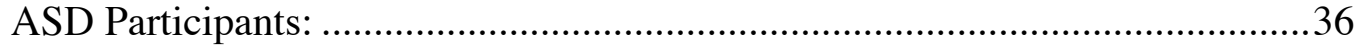

Movement Time (MT) of Participants with ASD ...........................................36

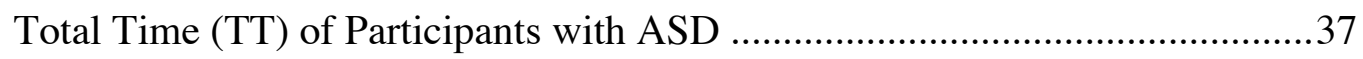

Dwell Time (DT) of Participants with ASD ................................................. 37

Percent time-to-peak velocity (\% TPV) of Participants with ASD ....................37

Participants who are Neurotypical: ............................................................ 38

Movement Time (MT) for Participants who are Neurotypical ........................38

Total Time (TT), Dwell Time (DT), and Percent time-to-peak velocity (\% TPV) for Participants who are Neurotypical .........................................39

Analysis including all participants: .......................................................... 40

Movement Time (MT) .........................................................................40

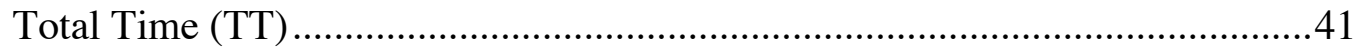

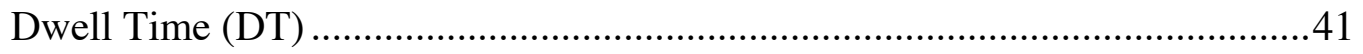

Percent time-to-peak velocity (\% TPV) ..................................................4 42

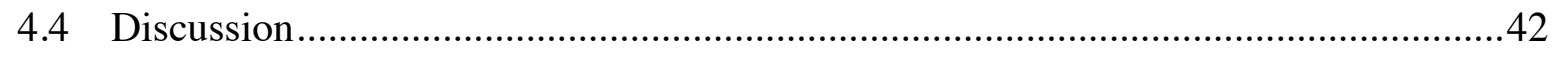

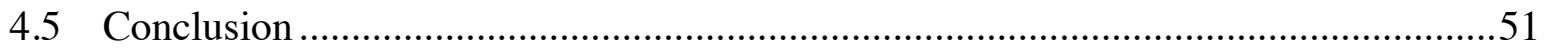

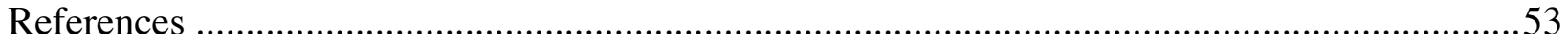

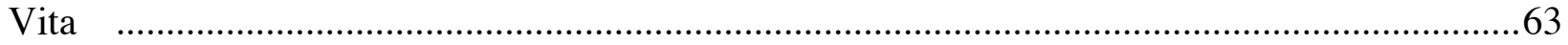




\section{List of Tables}

TABLE 1.1: $\left(\mathrm{TOL}^{\mathrm{DX}}\right): 2^{\text {nd }}$ Edition Participant Classification Scores ..............................................32 


\section{List of Figures}

Figure 1: Decreased cerebellum size in an individual with ASD compared to an individual who is NT. Adapted from Palmen et al. (2004)

Figure 2: Typical velocity and typical acceleration curves of neurotypical children in a goal directed movement

Figure 3: Tower of London-Drexel University (TOL $\left.{ }^{\mathrm{Dx}}\right): 2^{\text {nd }}$ Edition .27

Figure 4: Custom-built arm bar system used to collect kinematic data .28

Figure 5: Visual depiction of the training protocols (Fitts and Sine Wave) from the Test 1 to training to Test 2 .

Figure 6: Limb displacement (purple) and velocity trace (red) in Test 1, training, and Test 2 of a single participant with ASD. Red dots on the displacement trace represent points of peak velocity; Blue dots represent movement termination and green represent movement initiation.

Figure 7: Mean movement time, Total time, Dwell time, and \% time to peak velocity of All

Participants with ASD. .36

Figure 8: Mean movement time, Total time, Dwell time, and \% time-to-peak velocity of All Neurotypical Participants.

Figure 9: Mean movement time, Total time, Dwell time, and \% time-to-peak velocity of All

Participants (ASD group and NT group)

Figure 10: Total time and Mean movement time of All Participants

(ASD group and NT group).

Figure 11: Total movement time, Dwell time, and \% time-to-peak velocity of each participant of the ASD group in the Sine Wave condition. 


\section{Chapter 1: Introduction}

\subsection{What IS AUTiSM SPECTRUM DiSORDER (ASD)?}

Autism Spectrum Disorder (ASD) describes a general diagnosis of a group of complex disorders of brain development (American Psychiatric Association, 2013) including autistic disorder, pervasive developmental disorder-not otherwise specified (PDD-NOS), childhood disintegrative disorder, and Asperger syndrome (Diagnosis, Causes \& Symptoms, 2016). Where the latter is associated with high levels of intellectuality and functionality (Landrigan, 2010).

Furthermore, ASD is a wide-spectrum disorder, meaning that its diagnosis is extremely variable among individuals; thus, no two diagnoses will be exactly alike because each person has the potential to be at a different level on the spectrum. Despite differences on clinical profiles, people with ASD share three common characteristics and therefore these neurodevelopmental disorders are mainly characterized by deficits in verbal and nonverbal communications, social interactions, and restrained, monotonous patterns of interests, activities, or behaviors (American Psychiatric Association, 1994, 2013; Brambilla et al., 2003; Isaksen et al., 2013; Schaafsma \& Pfaff, 2014; Wing, 1997). In addition, people with ASD experience additional challenges in sensory processing, organization and attention, cognitive impairment, motor challenges, and emotional issues.

Moreover, ASD may also appear with conjunct physical and medical issues such as genetic disorders, sleep disturbances, poor nutrition, and/or mental health disorders (Sukhodolsky et al., 2008). These characteristics may become noticeable at early months after birth or later depending on the severity of the diagnosis, which ranges from mild to very intense (Itzchak, Lahat, \& Zachor, 2011; Landrigan, 2010). Research has indicated that there are no specific causes for ASD but there are valid theories that together could justify its development. 
These theories refer to abnormalities of brain structure and function, genetics, heredity, and possible medical problems (e.g. Allen et al., 2005; Bailey et al., 1995; Bolton et al., 1994; Freitag, 2007; Whitehouse, 2016; Wing and Potter, 2002).

\subsection{Prevalence and Costs}

ASD is one of the most widespread and prevalent developmental disorders in the United States with recent estimates of 1 in 68 children being diagnosed (Christensen et al., 2016). The prevalence of ASD is higher in boys, occurring one in 42 when compared to girls, which occurs at a rate of one in 189 (Christensen et al., 2016). Intellectual ability also varies in ASD; estimates of $43.9 \%$ of children ranked average or above average intellectual abilities with an IQ score greater than $85 ; 31.6 \%$ of children were classified with intellectual disability with an IQ score lower or equal to 70 ; and $24.5 \%$ of children were in the borderline with an IQ score between 71 and 85 . It was also reported that the percentage of intellectual ability among girls (37\%) was significantly higher than the percentage of intellectual ability for boys (30\%; Christensen et al., 2016). Estimates also reveal a difference in prevalence among different ethnic groups. Christensen et al. (2016) reported that ASD occurs significantly more among white non-Hispanic children (one in 64), than in black non-Hispanic children (one in 75), Asian children (one in 88), and Hispanic children (one in 99).

The estimated economic burden of ASD in the United States for the year 2015, including direct and indirect costs, was $\$ 268$ billion (Leigh \& $\mathrm{Du}, 2015$ ). Expecting this amount to increase every year, economic estimates for the year 2025 approximates to $\$ 461$ billion (Leigh \& $\mathrm{Du}$, 2015). In comparison, the estimated cost of ASD in the year 2015 was similar to those of diabetes and attention deficit and hyperactivity disorder (ADHD) and exceeded those of stroke and hypertension (Leigh \& $\mathrm{Du}, 2015$ ). If the prevalence of ASD continues to expand, it is likely 
that by the year 2025 the costs of ASD are going to exceed those of diabetes and ADHD as well.

\subsection{MOTOR IMPAIRMENTS IN CHILDREN WITH ASD}

In the seminal work of Kanner (1943), individuals with ASD were primarily described with stereotyped behavior and deficits in emotional gestures, socialization, and communication. In addition, the majority of these individuals' motor milestones were described by Kanner to be "normal" with proficient motor coordination, but others were described to have motor impairments. Not long after, Asperger's early observations of movement organization and performance in children diagnosed with ASD indicated that they were clumsy, uncoordinated, and had problems in writing and drawing (Asperger, 1991, 1944). In a descriptive study by Wing (1981), 34 cases of individuals between the ages of 5 - 35 years were analyzed in terms of Asperger's descriptions of ASD. The majority demonstrated poor motor performance such as an inability to run or walk smoothly, and also executive problems that were reflected in writing and drawing. As impactful as these observations were many years ago, they did not receive much attention until recently, when motor behavior in this population became a primary focus of research because it is thought to be strongly associated with the core symptoms of ASD.

\subsubsection{Gross and Fine Motor Impairments}

Although traditionally defined by repetitive behaviors as well as social and communicative deficits, new evidence has shown that ASD is linked to distinct motor impairments that can be classified as gross or fine in nature. A meta-analysis by Fournier, Hass, Naik, Lodha, and Cauraugh (2010) included 83 studies related to motor coordination, reach and grasp, gait, and postural control impairments where children with ASD in comparison to children who are neurotypical (NT), performed significantly different across all behaviors. In another study, Provost, Lopez, and Heimerl (2007) examined the following motor skills: stability, 
locomotion, object manipulation, grasping, and visual motor integration of children between the ages of $2-3$ months diagnosed with ASD, developmental concerns, and developmental delays. The authors used two standardized tests, the Bayley Scales of Infant Development II Motor Scale and the Peabody Developmental Motor Scales, $2^{\text {nd }}$ Edition. All children with ASD displayed delays in gross motor skills, fine motor skills or both. In comparison to children with developmental concerns, children with ASD showed significant motor impairments, but not when compared to children with developmental delays as both groups were not within the normal limits of the tests. Vilensky, Dmasio, and Maurer (1981) reported unusual gait patterns in children with ASD between the ages of $3-10$ years following a gait analysis. The children showed reduced stride lengths, increased stance times, increased hip flexion in the toe off phase, and decreased knee extension and ankle dorsiflexion during the ground contact phase. Other studies that analyzed posture, found that children with ASD have limited postural control and exhibit decreased postural stability, increased sway, and abnormal weight distribution in different stance positions (Minshe, Sung, Jones, \& Furman, 2004; Kohen-Raz, Volkman, \& Cohen, 1992; Molloy, Dietrich, \& Bhattacharya, 2003; Fournier et al., 2010). In addition, some studies analyzed postural control by removing visual input, resulting in a significant increase in postural sway. Fournier et al. (2010) and Molloy et al. (2003) proposed that individuals with ASD have somatosensory impairment. Since postural control is essential to perform more complex movements such as walking, it is no surprise that these children display gait impairments.

\subsubsection{Motor Planning and Control Impairments}

Motor deficits in individuals with ASD have also been shown to alter motor planning and execution of movements (Dowd, McGinley, Taffe \& Rinehart, 2012; Rinehart et al., 2006). To 
further explain, planning processes control the initial phase of the movement (e.g. reaction time, peak velocity) and corrective processes control the final phase of the movement (e.g. movement time, deceleration; Meyer et al., 1988). Dowd et al. (2012) examined children diagnosed with ASD between the ages of $3-7$ years performing an aiming task. They analyzed the upper limb movement kinematics of the children's performance and when the ASD group was compared to the NT group, they displayed significant variability in their movement preparation suggesting motor planning impairments. Conversely, Rinehart et al. (2006) also studied individuals with ASD between the ages of $6-20$ years performing an aiming task. The task consisted of moving as fast as possible to one of two targets that were set at equal distance in front of the participant, one over the left side and the other over the right side. A light was positioned on top of each target to serve as a visual queue for movement direction. When compared to the NT group, individuals with ASD displayed difficulty in adjusting the movement by spending longer time in the decelerating phase. These data suggested that they struggled regulating the final phase of movement to accurately approach the target. This topic will be discussed further in Chapter 3 .

\subsubsection{Gestures and Motor Imitation Impairments}

Gestures and motor imitation have also been found to be impaired in individuals with ASD. Rogers, Hepbum, Stackhouse, and Wehner (2003) found that children diagnosed with ASD at the age of 34 months could not imitate manual, facial, and object oriented tasks in comparison to both children who are NT and children with other developmental disorders in general. In addition, researchers have linked a possible association of motor ability and imitation impairments (Van Vuchelen, Roeyers, \& De Weerdt, 2007; Green et al., 2009). For instance, Van Vuchelen et al. (2007) examined high functioning and low functioning children with ASD at the age of 6 years performing various imitation tasks. The children were asked to imitate 
different gestures as well as different hand postures. When their performance was then compared to the children who are NT, the children with ASD not only displayed greater errors in the imitation performance, but also demonstrated increased variability in their hand postures. Other studies have also associated motor performance with intelligence quotient (IQ; Green et al., 2009; Mari, Castiello, Marks, Marrafa, \& Prior, 2003). For example, Green et al. (2009) assessed motor skills of children with ASD between the ages of $10-12$ years, using the Movement Assessment Battery for Children. The authors found a correlation between motor performance and IQ level, that is, individuals with lower IQ levels demonstrated poorer motor performance when compared to the individuals with higher IQs.

\subsubsection{Early Motor Impairments}

A number of studies have found that motor deficits have the potential to present themselves at an early age and, although hypothetical at this point, the strengthening of literature on ASD kinematics has great potential to establish a set expectation for early diagnosis (e.g., Adrien et al., 1993; Baranek, 1999; Bhat, Galloway, \& Landa, 2012; Landa \& Garrett-Mayer, 2016; Teitelbaum et al., 1998, 2004). In a prospective study, gross motor behavior was assessed in infants between the ages of 3 and 6 months with high-risk of developing ASD using the Alberta Infant Motor Scale. The children were also assessed for their communication level at 18 months of age with the Mullen Scale of Early Learning (MSEL). When compared to the NT group, the high-risk infants demonstrated motor impairments at 3 and 6 months of age, and those who experienced communication delays at 18 months of age also exhibited motor delays at 3 months of age (Bhat, Galloway, \& Landa, 2012). Landa and Garrett-Mayer (2006) examined low-risk and high-risk infants of developing ASD with the MSEL at 6 and 14 months of age. There were no significant differences between the groups at 6 months of age; however, infants 
that were later diagnosed with ASD began to show delays in motor development at 14 months of age, and by 24 months motor development was significantly delayed.

Various retrospective studies analyzing home videos of children younger than 3 years who were later diagnosed with ASD showed differences from a control group in sensory-motor deficits, lower muscle tone, decreased motor activity, retained primitive reflexes, and unusual postures (Adrien et al., 1993; Baranek, 1999; Teitelbaum et al., 1998, 2004). In contrast, Ozonoff et al. (2008) did not find early movement abnormalities in infants that were diagnosed with ASD when compared to the NT group. Similarly, Loh and colleagues (2007) reported that the stereotyped behaviors found in infants diagnosed with ASD did not differ from the NT group. Thus, these authors suggest a more comprehensive evaluation for early diagnosis of ASD is needed.

According to Ming, Brimacombe, and Wagner (2007) all the motor irregularities experienced by children with ASD are classified as "associated symptoms" and are believed to obstruct the development of other adaptive skills (Bauman, 1992; Leary \& Hill, 1996); implying that motor functionality may provide children the confidence to independently perform activities of daily living tasks. They further suggest that any abnormalities could negatively affect children's performance of activities of daily living and interaction with others.

\subsubsection{Cerebellum Dysfunction}

Based on the linkage of motor impairments to ASD, it should come as no surprise that a neurobiological explanation has evolved in the literature. Given the cerebellum's role in motor learning, controlling movement, coordination, and cognitive processes (Sivaswamy et al., 2010), an emerging theory of cerebellum dysfunction has become a leading candidate for explaining motor impairments in children with ASD. The cerebellum is described as the most consistent 
anatomical abnormality in the brain of people with ASD (see review by Allen et al., 2005). The cerebellum in children with ASD presents a smaller posterior lobe surface area (see Figure 1) as well as a decrease in Purkinje cells activity, a major output of the cerebellums neural control to the brainstem. Considering the constant interaction between the peripheral and central nervous systems to manage sensory input, integration, and motor output functions the chance of introducing errors when executing a movement is great (Mugnaini, 1972).

\section{Structural (MRI) Brain Imaging}

\section{Autism: 16y male Neurotypical: 16y male}
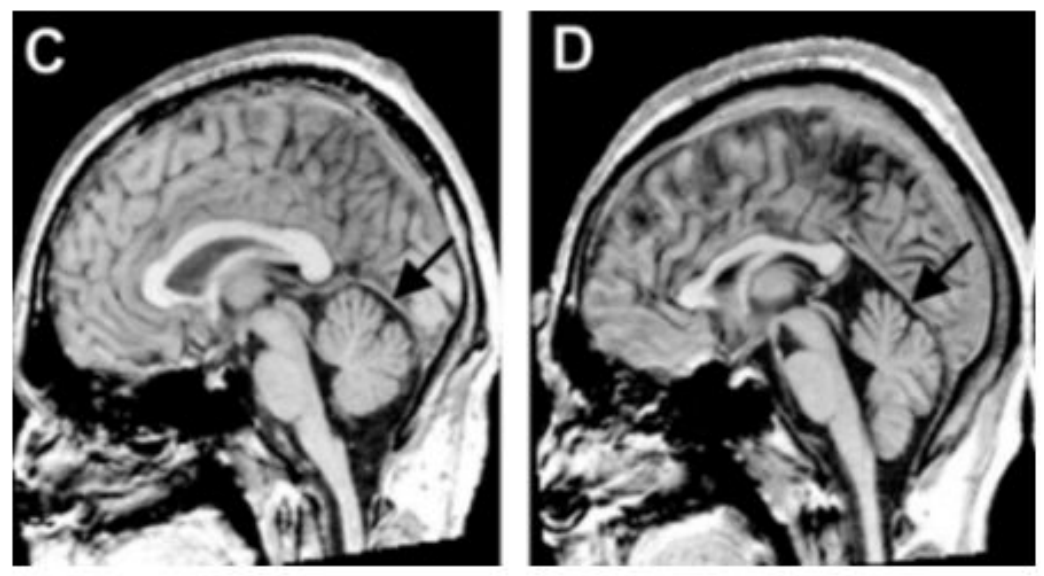

Figure 1: Decreased cerebellum size in an individual with ASD compared to an individual who is NT. Adapted from Palmen et al. (2004)

A number of studies have associated motor impairment performance with cerebellar dysfunction. Freitag et al. (2007) used the Zurich Neuromotor Assessment (ZNA) to examine motor impairments in children with ASD between the ages of $14-20$ years. The ZNA assesses simple and complex movements, performance of dominant and non-dominant extremities, and other motor components. The results indicated impaired balance skills and diadochokinesis (the ability to make antagonistic movements in quick succession). These findings along with other 
studies that reported postural control problems, suggest impairments in interaction and integration of sensory and motor functions. Moreover, these problems reflect the typical motor impairments that require coordination of alternating legs and arms and rapid antagonistic movements, similar motor problems are usually related to cerebellar dysfunction (Freitag et al., 2007; Allen et al., 2005).

A study conducted by Campione, Piazza, Villa, and Molteni (2016) investigated whether impairments in motor execution were associated with feed-forward or feedback-based mechanisms. A feed-forward process involves the planning or programming prior to a movement, while feedback relates to the integration and modification of motor skill execution based on sensory input. Children diagnosed with ASD between the ages of $4-5$ years were examined performing a reach and grasp task. The participants were instructed to reach for a small or a big object while the kinematics of their movements were recorded. In comparison to the NT group, the results indicated that the children with ASD exhibited longer reaching times reflecting higher duration of the feed-forward phase of the movement and less duration in the deceleration phase reflecting the feedback processes during the movement. The authors concluded that the ability to modulate the reaching kinematics of the movement were impaired, because children spent more time in the acceleration phase of the movement suggesting problems with feed-forward processing of information (Campione et al., 2016).

Although research to better understand motor impairments in people with ASD is growing, additional study is needed to more precisely examine and identify the unique kinematic characteristics of movement behavior in children with ASD while performing a reciprocal task, and examine novel interventions to address any inefficiency or adaptability in the control of motor skills. The present study examined the planning, execution, and homing-in phase of a 
reciprocal goal directed upper extremity movement as well as the adaptability of tracing an optimized template to enhance coordination in children with ASD.

With regard to the motor control literature, a widely used assessment for motor planning and execution is reflected through a mathematical equation by establishing the level of difficulty of a goal directed movement. In the following section, a more detailed description of this equation, its evaluation, and its relevance to people with ASD will be provided. 


\section{Chapter 2: Goal Directed Movements}

\subsection{FitTs LAW}

For over 100 years, Woodworth's work has been fundamental in studies examining goal directed movements of the upper extremities. R.S. Woodworth (1899) studied movement characteristics of goal directed movements by having participants draw a horizontal line between two targets of a fixed distance. The speed and movement time (MT) were adjustable which allowed Woodworth to examine the accuracy and consistency of movements around the target points as well as spatial and temporal characteristics of movement trajectories as the difficulty changed. Following his study, Woodworth concluded that goal directed movements are composed of two distinct phases; an initial projection phase dependent on planning processes, that brings the limb close to a target, and a late phase dependent on control, also called feedbackbased processes (Woodworth, 1899). This second phase allows the participant to process sensory information such as visual and proprioceptive feedback where, if necessary, adaptive corrections occur in order to increase accuracy of hitting the target. Although influential then as well as today, Woodworth's findings were not implemented until the 1950's.

The seminal work of Paul Fitts revealed that when participants strived to move between targets, increases in movement distance (D) and/or reduction in target width (W) resulted in longer average movement time (MT) as a result of increased attention demands (Fitts, 1954). Along with Shannon's information theory (1949), Fitts developed an index of difficulty (ID), which he theorized was a result of the number of bits of information to be processed to efficiently generate the desired level of precision required to successfully move between the targets. Although variations are present today, one of the most widely used calculations of ID can be determined by the equation $\log _{2}(2 \mathrm{D} / \mathrm{W})$, where $\mathrm{D}$ represents the distance of the 
movement measured from one target center to the other, and $\mathrm{W}$ represents the corresponding width of the target. Additionally, MT across a range of IDs can be characterized by the equation $\mathrm{MT}=\mathrm{a}+\mathrm{b}$ (ID).

Goal directed movement of the limbs, whether reciprocal (Adam \& Paas, 1996; Boyle \& Shea, 2011; Guiard, 1997; Kovacs, Buchanan, \& Shea, 2008; Mottet \& Bootsma, 1999) or discrete (Fitts \& Peterson, 1964; Meyer et al., 1988) traditionally exhibit a speed for accuracy trade-off as difficulty increases (Fitts, 1954; Woodworth, 1899). In other words, as the difficulty of the task increases, performers must decrease movement time in order to accurately strike the target area. In relation to the control processes and kinematic variables related to this type of task, studies have consistently revealed that as movement time decreases, the proportion of time spent in the acceleration stage of the movement decreases. This unequal shift in movement components indicates that as difficulty increases, movement control shifts from preplanned, more cyclical control to online, more discrete control (e.g., Buchanan, Park, \& Shea, 2006).

Meyer et al. (1988) suggested that goal directed movements consist of an initial and a secondary phase, each operating under different control processes. The initial phase is preplanned, while the secondary phase is controlled during the execution of the movement using visual and proprioceptive feedback. Spatial and temporal measures of movement trajectories are used to identify if a movement is pre-planned or controlled (Elliot, 2010). These characteristics are defined by displacement and time profiles that are used to calculate velocity. Velocity is then used to calculate acceleration. Discontinuities in the velocity or acceleration profiles are used to identify the onset of feedback-based regulation (Elliot, 2010). The symmetry of both velocity and acceleration profiles along with any discontinuities in the movement trajectory are useful to understand how a movement is planned or controlled (see Figure 2; Thomas, Yan, \& Stelmach, 
2000). For instance, the movement-planning process is associated with movement initiation time and early trajectory characteristics (Meyer et al., 1988). Whereas the control process is associated with later trajectory characteristics along with discontinuities in velocity and acceleration patterns (Walker, Philbin, Worden, \& Smelcer, 1997). Similar to Woodworth's twocomponent model of limb control, recent studies have suggested that the initial movement phase is an online movement dependent on motor planning processes (Beggs \& Howart, 1970; Carlton, 1981; Elliott, Hansen, Mendoza, \& Tremblay, 2004; Meyer et al., 1988). That is, as the movement begins to unfold the pre-programmed movement estimates the expected movement of the limb.

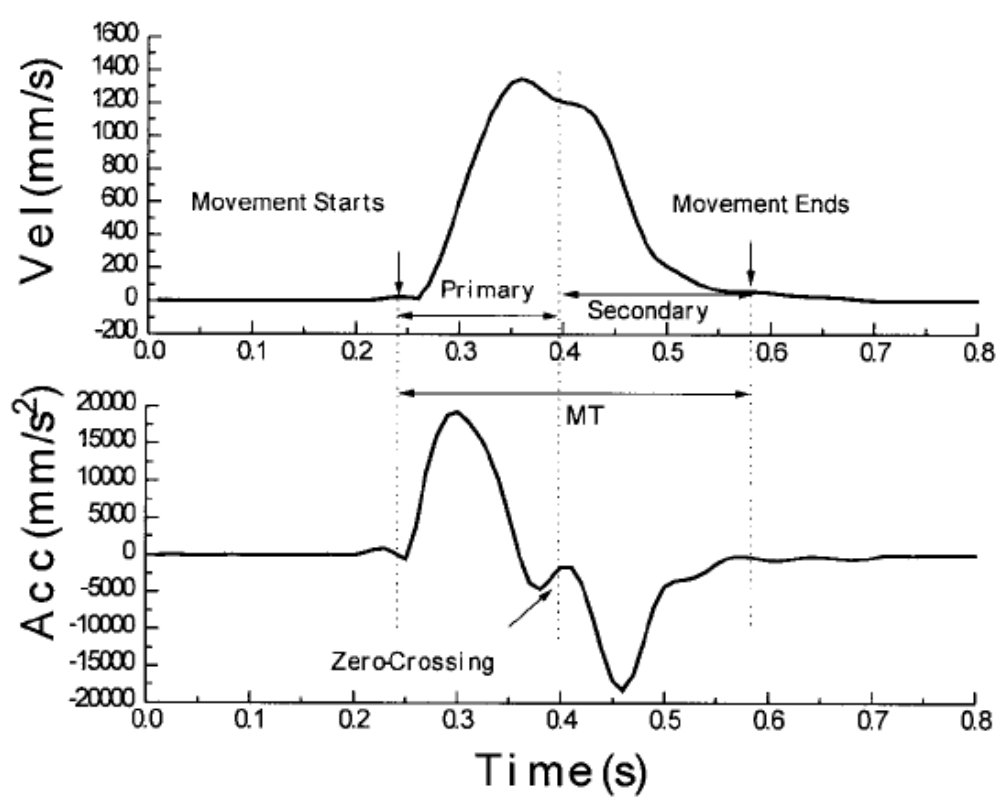

Figure 2: Typical velocity and typical acceleration curves of neurotypical children in a goal directed movement

\subsection{Novel Sine WAVE Tracing Task}

Although a number of studies have examined performance improvements with repetition in the Fitts paradigm (for review see Elliott et al., 2010), it is worth noting that after practicing 
over a period of 20 days a variety of Fitts protocols using different IDs, participants showed very little improvement in endpoint variability (Boyle, Panzer, Wright, \& Shea, 2012a; Kelso, 1995). Boyle et al. (2012a) suggested that due to frequent performance of aiming tasks in activities of daily living, a movement strategy has developed that is highly effective and can no longer be improved when practiced using a Fitts task. However, this conclusion is assumed disregarding the use of augmented perceptual information, which typically leads to movement enhancement (Kovacs et al., 2008). More recently Boyle et al. $(2012 b, 2014,2015)$ have shown that through tracking a novel template, performance on a reciprocal Fitts task can be enhanced by adding information that forces the participant to adopt a different movement strategy than would typically be used, ultimately leading to enhanced performance. This suggests that if performance on a reciprocal Fitts task which encompasses goal directed movements of the limbs can be enhanced, then performance of aiming tasks related to activities of daily living also has the potential to be improved by using template tracking.

A recent study by Boyle and colleagues (2012b) showed that after participants traced a sine wave template by flexing and extending their right arm in the horizontal plane, their performance on a post-test Fitts target task improved. Although Fitts law does not negate faster movement times per se, it does predict that faster movement times would occur at the cost of movement accuracy (Fitts, 1954). Boyle et al. showed that his was not the case. Not only did participants make faster movement times on the Fitts task following sine wave tracking practice, but these movements were constructed of smoother velocity profiles, lower dwell time in the target space, and a reduction in corrective sub-movements (Boyle, Kennedy \& Shea, 2012b). These results suggested that the use of the template during practice enhanced the participant's 
ability to adapt their motor program in a way that was not dependent on stimulus availability during the homing-in phase of the movement (Kovacs and Shea, 2011).

The specificity of learning hypothesis suggests that skill performance during testing deteriorates when sensory information used during practice is removed, because participants tend to become dependent on it (Coull, Tremblay, \& Elliot, 2001; Debaere, Wenderoth, Sunaert, Van Hecke, \& Swinnen, 2003; Proteau, 1995; Schmidt \& Wulf, 1997). Attempting to diminish this dependency and to improve performance, Kovacs and Shea (2011) found that reduced frequency of augmented feedback displays is an effective way to reduce participants' dependency on the feedback provided during practice. Using the sine wave template during practice, Boyle et al., (2012b, 2014, 2015) showed that participants in the sine-wave condition did not exhibit any performance decrement on a high ID Fitts task after using the template to guide their performance during acquisition. These results clearly contradict the specificity of learning hypothesis, however, the participants using a cyclical control rather than a discrete control or following the sine wave to guide their movements, could explain the divergence of the results with previous research.

Following the 2012b study, a series of investigations examined the role of the sine wave with respect to the size of the template (Boyle, Kennedy, Wang, \& Shea, 2014), the role of the performer versus observer (Boyle, Kennedy, Wang, \& Shea, 2016) and the effect of aging (Boyle, Kennedy, \& Shea, 2015). Regarding the aging question, the authors noted that the specific enhancements seen following the sine wave tracking task, i.e. faster movement times with retained/enhanced smoothness, were critical pieces consistently described as areas of decrease in elderly goal directed movements (Ketcham, Seidler, Van Gemmert \& Stelmach, 2002). Interestingly, these upper movement impairments presented in the elderly seem to also 
occur in the people with ASD (see review Sacrey, Armstrong, Bryson, \& Zwaigenbaum, 2014). Boyle et al. (2015) assessed young and elderly participants on a Fitts pre-test task using 15 trials, and then each participant group was subdivided in to a sine wave training group or a Fitts target task control group. Following 45 trials of practice in their respective group, all participants performed a 15-trial Fitts post-test task. The results from the study indicated that the elderly did in fact display inferior performance compared to both young adult conditions in the Fitts pre-test. However, following the acquisition phase with the sine wave tracking task, the elderly participants produced similar movement patterns compared to the young adult Fitts control group. Although the elderly did not improve to the level of performance of the young participants in the sine wave condition, they did performed similarly to the young adults who performed the Fitts target task the entire time. These results support the conclusion that a novel task of fast and harmonic motion has the potential to alter what seemed to be rigid movement strategies not only in young adults, but also in populations that display irregular goal directed movements, such as the elderly.

As a result of the findings reported by Boyle et al. (2015), it is believed that children with ASD could possibly benefit from tracing the sine wave in a similar way as the elderly did to improve their performance in goal directed movements. 


\section{Chapter 3: Movement Structure of Children with ASD}

\subsection{Motor Planning ANd CONTROL}

Movement behavior is composed of movement skills and motor abilities. Movement skills are movements with a specific purpose such as locomotion and object control. These are motor skills needed to move or receive an object with accuracy and control (Burton \& Miller 1998). Thus, movement skills are considered the foundational movements for the more advanced skills required for play or sports (Burton \& Miller 1998). On the other hand, motor abilities refer to the capacity to perform a movement skill. Motor abilities cannot be observed directly but are rather inferred from the movement kinematics of the individual's performance (Magill, 1998).

Research has consistently associated people with ASD with motor impairments and poor movement skills, thus indicating poor motor abilities as well (see review Sacrey et al., 2014; Staples \& Reid, 2010). In the following sections movement behavior of upper limb goal directed movements in people with ASD is going to be further explained.

\subsubsection{Motor Planning Impairments}

Glazebrook, Elliot, and Lyons (2006) examined young adults with ASD and young adults who are NT between the ages of 20 - 30 years, who performed rapid discrete aiming movements to one of two targets. Following the Fitts (1954) paradigm, different levels of movement difficulty were created by modifying both target size and distance. When compared to the NT group, participants with ASD displayed more spatial and temporal variability in the first phase of the movement, indicated by the movement time at the different indices of difficulty, but were able to maintain end-point accuracy. The ASD group also spent more time preparing and executing movements. Thus, the authors suggested that young adults with ASD try to reduce spatial variability that occurs in the online control phase by learning to adjust their initial 
impulses. Dowd et al. (2012) reported similar results in children with ASD between the ages 3 7 years when they performed a point-to-point aimed task. They analyzed the time kinematics of the children's performance and found that the ASD group displayed substantial variability in their movement preparation compared to the NT group, suggesting motor planning impairments.

A follow-up study by Glazebrook, Elliot, and Szatmari (2008) attempted to investigate how motor performance of a rapid manual aiming task was affected when information was provided in advance in a direct manner. The authors examined participants with ASD between the ages of $20-24$ years performing rapid aiming movements using a box with ten buttons. Of the 10 buttons two were white, indicating starting positions, and the others were illuminated with a red light, indicating a pre-cue sign for which hand to use (left or right), and in which direction to move (left or right) to reach the target. For example, a pre-cue illuminating the four buttons to be controlled by the right or the left hand specified both the direction to move and the hand to use. Participants with ASD showed similar reaction times as the NT group, however, overall they took longer to start the movement and displayed more variability in the starting phase of the movement. Aside from this, the results indicated that young adults with ASD benefited from the pre-cues to perform overall faster movements. That is, they were able to take advantage of advance information to construct their motor plan.

Similar to the previous study, Nazarali, Glazebrook, and Elliott (2009) examined how young adults diagnosed with ASD at the age of 26 were able to plan and reprogram their movements. The task included a box with six buttons, where two of the buttons were considered the starting positions where participants placed their index finger and reach to the designated target as indicated by the pre-cue. The buttons were illuminated to indicate the pre-cue of which hand to use or which direction to move. For example, direction was indicated when the left or 
right button next to the left/right index finger was illuminated and to determine which hand to use, both buttons next to the index finger were illuminated. Once the pre-cue was delivered, the light turned off and the target button was illuminated. In this condition, the participants with ASD were able to use the pre-cue information and perform their movements with similar timing as the NT group, but they still took longer overall. These findings supported the results reported by Glazebrook et al. (2008). The second condition of the study was similar to the one previously described, but a false queue was included. That is, the pre-cue button was illuminated indicating either a left/right hand or left/right direction, but the real target would be the opposite. This condition required that the participant had to rearrange their movement plan. In this condition, when the false queue was delivered, participants with ASD took longer to reprogram and execute their movements. More specifically, it took them more time to reprogram which hand to use than in which direction to go.

When analyzing how children with ASD perform reach and grasp tasks, Fabbri-Destro, Cattaneo, Boria, and Rizzolatti (2009) reported that children seemed to plan sequential movements in separate or independent steps. In their study, participants with ASD aged 10 years, were required to reach and grasp a metal object and place it into one of two containers. The containers were of different sizes, small or large. These containers were randomly switched to see if there were any differences in motor planning execution. The kinematics of the hand movements indicated that children with ASD did not show an adjustment in time through the reaching phase similar to the NT group when the level of difficulty of the task was changed. This result indicated that they planned their movements without considering their final target. Similar to the previous study, Hughes (1996) attempted to investigate the reach and grasp performance of children with ASD between the ages of $12-14$ years. Where they concluded that children 
with ASD struggle to plan their movements in a sequential fashion. The participants were studied performing a reach, grasp, and place task. The task consisted of reaching for a rod (half painted in black and half in white), grasping it, and transporting it to a disk sitting on a tabletop. They then had to stick either the white or black end of the rod in the disk. The correct way to perform the task was by grasping the rod with an uncomfortable handgrip position and finishing in a handgrip comfortable position. In comparison to the NT group, children with ASD did not make corrections to their handgrip in order to finish in a comfortable position. Based on the results, the authors concluded that children with ASD are not able to plan a complete movement. Hughes' conclusion that children with ASD struggle to plan their movements in an effective sequential fashion, was supported by Cattaneo et al. (2007), who recorded muscular activation of the mouth using the electromyography (EMG) when performing an eating task. The NT group experienced muscle activation as soon as they began reaching for a piece of food. In contrast, children with ASD experienced muscle activation after the piece of food was grasped and the hand was nearing the mouth.

\subsubsection{Motor Control Impairments}

Mari et al., (2003) reported that children with ASD did not display problems with their motor planning in a reaching and grasping task, but that they displayed impairments in their homing-in phase. The authors examined children with ASD between the ages of $7-13$ years, who were instructed to reach and grasp a cube that was placed in front of them. The cubes varied

in size, large or small, and were placed near $(18 \mathrm{~cm})$ or far $(28 \mathrm{~cm})$ from the participant. The movement kinematics demonstrated that children with ASD performed the task much faster than the control group, suggesting that children with ASD developed a movement strategy because 
they were not capable of processing the concurrent visual feedback as they were executing the movement.

Forti et al. (2011) investigated whether motor abnormalities in children with ASD originated from motor planning dysfunction (also see Glazebrook et al., 2006) or from online feedback processing deficits (see Mari et al., 2003). Forti et al. (2011) required participants to reach for a ball that was placed on a table and transport it to a hole, which was inside of a seetrough box in which the ball was to be dropped. No variations in the initial movement impulse were found, however, participants with ASD showed higher velocities and additional corrective sub-movements in the homing-in phase. The additional sub-movements implied that children with ASD performed the homing-in phase with a less efficient structure, it did not interfere with achieving similar levels of accuracy compared to the control group. The presence of additional sub-movements in the secondary phase of the movement task suggested that children with ASD have feedback processing impairments and/or lack the capacity to create a complete motor plan prior to executing the movement.

Children with ASD are also believed to rely on proprioceptive feedback when executing movements (Schmitz, Martineau, Barthélémy \& Assaiante, 2003). Schmitz et al. (2003) examined children with ASD between the ages of $7-8$ years. The children were asked to hold a load on a small platform that was tied on their right hand. The experiment consisted of two conditions, in one the experimenter removed the load from the participant and in the other condition, the participant removed the load. Muscle activation of the biceps brachii was recorded and indicated that in neither condition, the children with ASD were able to anticipate load removal as the biceps brachii contraction was activated after sensory feedback was received. These results suggested that children with ASD were not capable of anticipating the change in 
load using visual cues. In other words, they were not capable of processing feed-forward information.

Studies by David et al. (2009) and David, Baranek, Wiesen, Miao, and Thorpe (2012) examined motor adjustments and showed that children with ASD seem incapable of using information from previous movements to correct their motor plan in advance and perform more effectively on subsequent trials. In the study of David et al. (2009), children with ASD between the ages of $10-11$ years were instructed to lift a weighted target and place it on a target platform. Grip and load force were measured during this grip-and-place task by a customized apparatus. In comparison to the NT group, participants with ASD demonstrated a delay in force onset to lift the target, greater grip force than necessary while transporting the target, and greater variability in their performance across trials. These results suggested that children with ASD were unable to use previous experience to anticipate lifting the load. In a follow-up study, the same protocol was used, but this time the performance of children with ASD was compared to children with developmental delays (David et al., 2012). Both groups exhibited late force onset and longer time to reach maximal grip force, suggesting that these motor impairments are not specific to ASD. These impairments could be the result from the lack of ability to process and respond to previous feedback, thus, relying on proprioceptive feedback every trial to complete movements (David et al., 2012). This finding suggests that children with ASD cannot use previous feedback to modify their motor plan, thus relying on concurrent feedback. It is important to note that the elderly, who suffer from similar upper movement impairments as the children with ASD, were capable of modifying their motor plan after tracking the sine wave (Boyle et al., 2015). 


\subsection{Motor Planning And Control Analysis Utilizing Fitts (1954) Paradigm}

Despite twenty years of research regarding motor function in ASD (e.g. Mari et al., 2003; Provost, Lopez \& Heimerl, 2007; Green et al., 2009; Sacrey et al., 2014), only a few studies have utilized the Fitts law speed-accuracy trade-off protocol (Glazebrook et al., 2006; Glazebrook et al., 2008; Papadopoulos et al., 2012). Glazebrook et al. (2006) examined young adults with ASD between the ages of $20-30$ years performing rapid discrete aiming movements to one of two targets that differed in levels of difficulty. The protocol consisted of placing the index finger in the middle of the two targets and as soon as one target circle was illuminated with a red light, the participant had to touch the target with their finger as fast and accurate as possible. Although participants with ASD were able to maintain end-point accuracy, their reaction time was greater than the NT group across all levels of difficulty indicating motor planning impairments. In a follow up experiment, Glazebrook et al. (2008) examined young adults with ASD between the ages of $17-30$ years. The authors wanted to know if they could use information that was not directly present in a goal directed movement. The protocol was similar to the one used by Glazebrook et al. (2006), but in this experiment participants were allowed to choose their starting position between the two targets knowing that either the left or the right target was going to light up. Participants with ASD took longer to initiate movement as well as complete it. Also, the authors concluded that the individuals with ASD were not influenced by the context of their movements, because the target size did not have an effect on their movement time.

A more recent study by Papadopoulos et al. (2012) investigated the kinematic composition of upper extremity movement of children with ASD between the age of 7 and 12 years performing a reciprocal manual-aiming task. The results showed no differences in movement time between NT group and children with ASD. Similar to the findings of Forti et al. 
(2011), participants with ASD showed more variability of movement during the homing-in phase of the task compared to the NT group. This could be explained by their difficulty to plan subsequent movements or by a lack of feed-forward control. An inability to use feed-forward is associated with a movement impairment present in patients with cerebellar injures (Rosenbaum, 1991), which could explain the difficulties in planning and regulating movements during the homing-in phase of a goal directed movement (Mosconi et al., 2015). 


\section{Chapter 4: Novel Approach to Enhancing Coordination in Children with ASD}

\subsection{INTRODUCTION}

Previous studies analyzing the movement kinematics of people with ASD have inferred that they suffer disturbances in the overall structure of their movements suggesting impairments in both cognitive processes, motor planning (Glazebrook et al., 2006; Glazebrook et al., 2008) and motor control (Mari et al., 2003; Papadopoulos et al., 2012; Rosenbaum, 1991).

Interestingly, it is the secondary phase of the movement, which is controlled by the motor control process, that after tracing the sine wave template has demonstrated the largest enhancement in the series of work by Boyle et al. (2012b, 2014, 2015).

Therefore, our study had several goals. First, we wanted to further the understanding of the kinematic composition of goal directed upper limb movements in children diagnosed with ASD. Specifically, we wanted to determine where in the construction of their movements we see differences in motor behavior compared to children who are NT. Second, we wanted to investigate whether children with ASD were capable of adapting limb movements after practicing a sine wave tracking task. This purpose is warranted given the overwhelming lack of investigations of this nature. We were especially interested in determining whether tracking a template that provided fast-harmonic motion, aided in the development of smoother velocity profiles in the homing-in phase of participants with ASD. This was considered especially important if participants with ASD show differences in movement kinematics compared to participants who are NT.

Two hypotheses were investigated in this study. First, participants with ASD will exhibit motor deficits in goal directed aiming movements, predominantly in the homing-in phase of the movement. Second, participants who practice tracking the sine wave template will exhibit 
improved kinematics during a reciprocal movement task (Fitts, 1954). More specifically, these improvements will likely be seen in faster overall movement time and a more harmonic construction of the entire movement pattern.

\subsection{METHOD}

\subsubsection{Participants}

Participants were children between the ages of $7-12$ years, recruited from the local community. The population consisted of nine children who are NT as well as ten children diagnosed with high functioning ASD. Typically developing children were also recruited from the local community. All participants were encouraged to visit the laboratory prior the testing session in order to become familiar with the facility. Due to the nature of the population, guardians were responsible for reading and signing the informed consent, and they were encouraged to take part in the instruction phase of the experiment. Guardians were also required to be present at all times. The guardians and participants were advised that if at any time the guardian or participant would like to leave the laboratory or terminate their participation in the experiment they were free to do so at no penalty to them.

\subsubsection{Apparatus}

Two apparatuses were used to collect data. First, initial executive planning was evaluated by the Tower of London-Drexel University $\left(\mathrm{TOL}^{\mathrm{DX}}\right): 2^{\text {nd }}$ Edition test (Culbertson \& Zillmer, 2011; see Figure 3), a neuropsychological instrument designed to assess executive planning abilities in children and adults. Two towers were placed on a table in front of each other, each with three beads on each peg. This position allowed the participants and the examiner to sit across from each other and have a tower in front of them where peg 1 of each tower mirrored the right hand of the participant and the left hand of the examiner. In this assessment, participants 
performed a total of 15 different exercises with the goal of matching the placement of the color beads from the tower of the experimenter in their tower. Before starting, participants were told that their goal was to copy the examiner's tower in the least amount of movements possible. They were also explained the two rules of the assessment: (1) they could only move one bead at a time and (2) they could not place more beads on the peg than the peg could hold. If they performed otherwise, it resulted in rule violation that required moving the beads back to where they were placed before committing the violation.

Seven dependent variables were recorded. Total Move represented the level of executive planning and supporting cognitive components. Total Correct measured working memory capacity and control. This variable allows mental move sequences to guide planning and problem solving. Total Rule Violation measured the ability of planning and problem solving with constraints, in this case, with rules. Total Time Violation measured the ability to plan and execute in a specific period of time. Total Initiation Time measured under- to over-controlled response processes. Slower initiation times are generally associated with a more thoughtful plan to problem solving. Total Execution Time measured how fast executive plans are solved. Total Problem-Solving illustrates overall executive planning as a function of problem-solving speed.

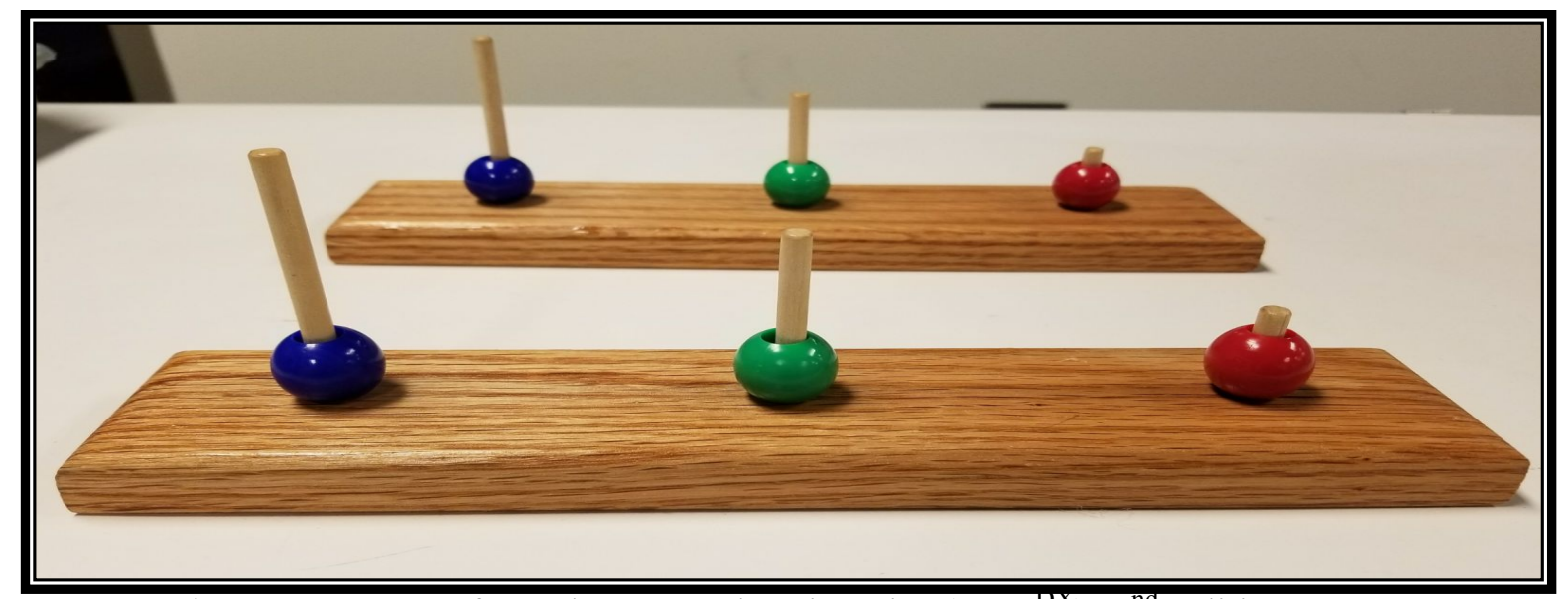

Figure 3: Tower of London-Drexel University $\left(\right.$ TOL $\left.^{\mathrm{DX}}\right): 2^{\text {nd }}$ Edition 
Second, a custom-built arm bar system was used to collect kinematic data of upper limb aiming movements (see Figure 4). The system was fastened to a table with adjustable tracks to position the arms in a comfortable position matching the participant's limb structure. The arms were constructed from aluminum, cushioned with foam padding and wrapped with a soft cloth material for comfort of the participants. A soft ergonomically designed grip was fastened at the end of the bars. The arms moved freely in the horizontal plane and were positioned with safety rods to prevent the arm bar from moving too close or far away from the participant. A height adjustable chair was also used to allow the participant an optimal posture for the execution of the arm movements.

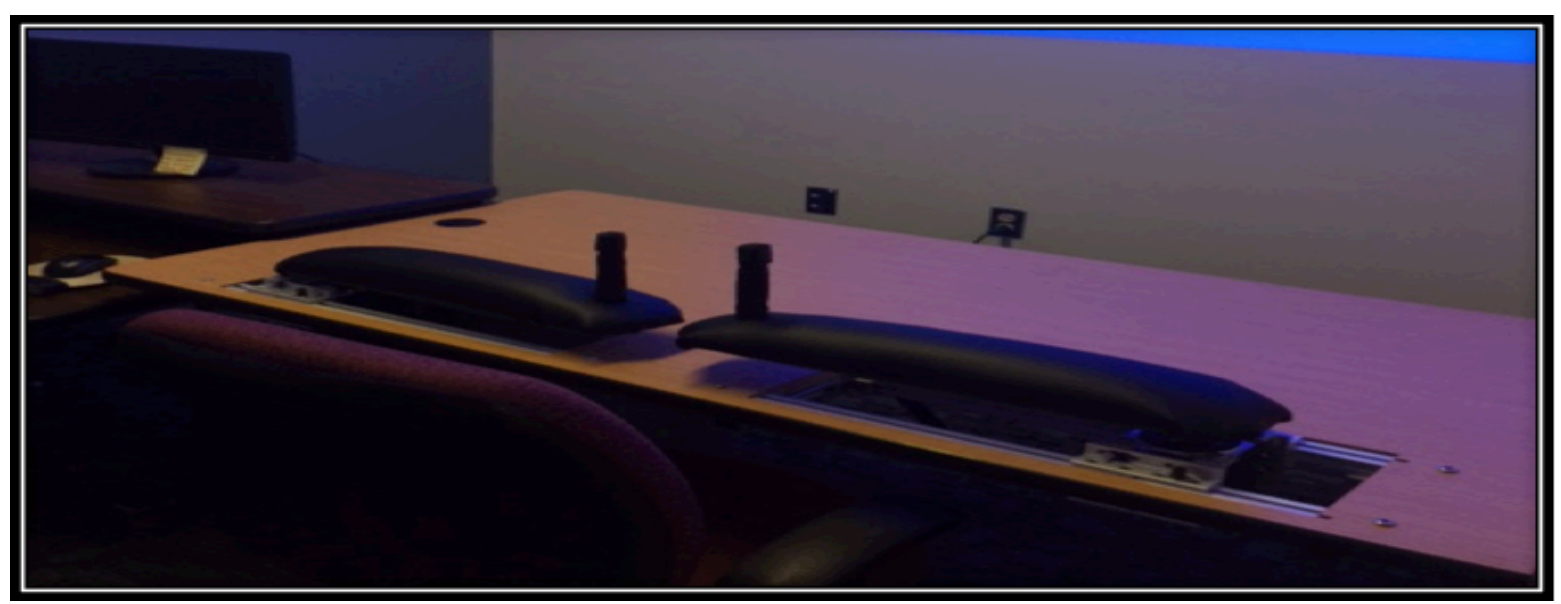

Figure 4: Custom-built arm bar system used to collect kinematic data

\subsubsection{Procedure}

Following the classification of the participants as ASD or NT, they were randomly assigned to a Sine Wave or Fitts training protocol (see Figure 5). In the Fitts-like reciprocal aiming task (Fitts) condition, a lab member demonstrated the task and its requirements. Following the example, the participants were seated with their right arm comfortably resting on the lever. A safety chair hugger was available in case it was requested by the guardian or 
participant. Participants were instructed to flex and extend the right lever in the horizontal plane and to move a cursor in and out of two clearly defined target areas on the screen in front of them. The participants were instructed to complete this task as quickly and accurately as they could. A single trial lasted 12 seconds and the participants were asked to complete a total of 15 trials. These 15 trials were analyzed as the baseline movement production and labeled Test 1. Following a five-minute break, the participants were asked to complete the same protocol for 30 trials during a training phase, and perform it again for 15 trials during Test 2. In total the participants completed 60 trials of the Fitts-like task.

In the Sine Wave condition, the Test 1 was completed in the same manner as in the Fitts condition (see Figure 5). After a five-minute break, the participants were asked to observe a lab member demonstrate the sine wave tracking task. Following the example, the participants were instructed to move the right lever by flexing and extending their elbow in order to trace the projected sine wave template on the screen. The participants were instructed to trace the sine wave to the best of their ability. A single trial lasted 12 seconds and the participants were asked to complete a total of 30 trials during the training phase. Following the sine wave tracking training phase, participants were asked to complete 15 additional trials of the Fitts task for Test 2. The procedure took an average of 45 minutes, however, a 2-hour window was set aside to ensure the participants remained as comfortable as possible during the session. 


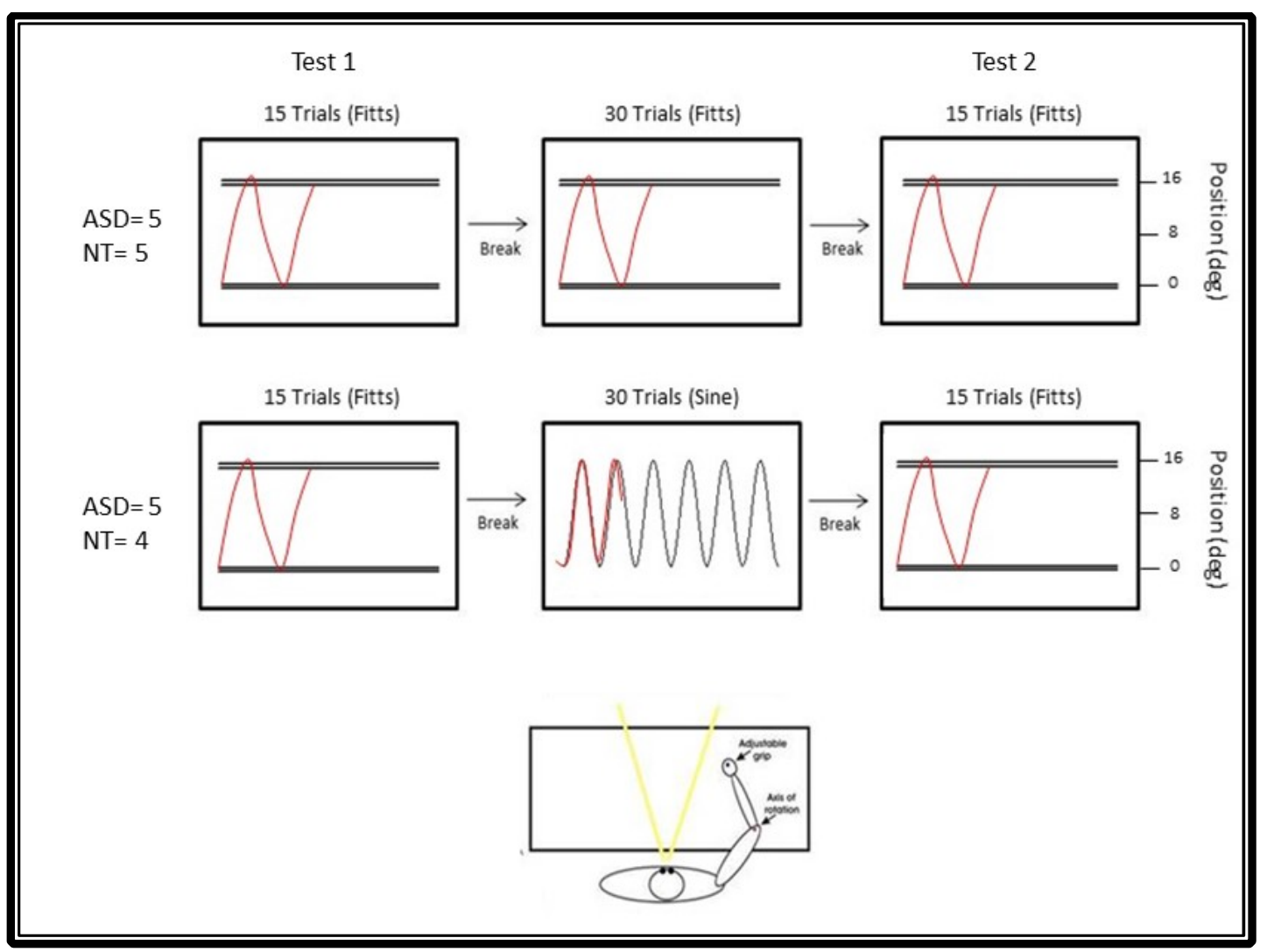

Figure 5: Visual depiction of the training protocols (Fitts and Sine Wave) from the Test 1 to training to Test 2.

\subsubsection{Measures \& Data Analysis}

\section{The TOL ${ }^{D X}: 2^{\text {nd }}$ Edition}

The TOL ${ }^{\mathrm{DX}}: 2^{\text {nd }}$ Edition (Culbertson \& Zillmer, 2011) variables were measured with the following calculations. Total Move was measured as the number of moves the participants made to match the placement of the beads from the examiner's tower. Total Correct was measured from the exercises that the participants completed with the least amount of movements according to the $\mathrm{TOL}^{\mathrm{DX}}$ score sheet. Total Rule Violation was measured with the total number of violations the participants made when performing the exercises. Total Time Violation was counted at oneminute mark after the exercise was started and at two-minute mark, the exercise was terminated. Total Initiation Time was the time past after telling the participants to try the exercise and when 
they first grabbed a bead. Total Execution Time was the time they took to solve the problem and finally, Total Problem-Solving was the sum of Total Initiation Time and Total Execution Time.

Each raw score from every variable for the Tower of London was standardized according to the general TOL ${ }^{\mathrm{DX}}$ guide (2011). These variables were averaged for the participants with ASD and for the children who are NT to assess the overall level of executive planning for each group. An independent T-test was conducted with an $\alpha=.10$ to see if any differences existed in the $\mathrm{TOL}^{\mathrm{DX}}$ variables between the performance of the two groups of participants.

\section{Kinematic Data}

The kinematic data were measured with a three-point central difference algorithm to calculate velocity of the limb. Dependent measures were analyzed on a half-cycle basis. Peak velocity (PV) and time of peak velocity (TPV) were determined during each half-cycle of limb movement. The onset of movement was determined by tracing backward from TPV to a value $2.5 \%$ of that half-cycle PV. Movement offset was calculated by tracing forward from TPV to a value $2.5 \%$ PV. For each half-cycle, there was a single movement onset, peak velocity, and movement offset. Total time of movement (TT) was calculated as the difference of the summation of movement onset plus one and movement onset. Movement time (MT) was calculated as the difference of movement offset and movement onset. Dwell time in the target at movement reversal (DT) was calculated as the difference of movement onset plus one and movement offset. Percent time-to-peak velocity (\%TPV) was calculated by the ratio of the difference of TPV and movement and the difference of movement offset and movement onset multiplied by 100 .

To better understand the individual groups, the dependent variables of MT, TT, DT, and $\%$ TPV were analyzed in separate Condition (Sine Wave, Fitts) $\times$ Test (Test 1 , Test 2 ) analyses of 
variance (ANOVAs) with repeated measure on Test. Additionally, the dependent variables of MT, TT, DT, and \%TPV were analyzed in separate Condition (Sine Wave, Fitts) $\times$ Group (ASD, NT) $\times$ Test (Test 1 , Test 2) linear mixed model analyses of variance (ANOVAs) with repeated measure on Test. Simple main effects analyses were utilized when appropriate as post hoc procedures to follow-up on significant interactions. Given the small population sample, an $\alpha=$ .10 was used for all tests.

\subsection{RESULTS}

A summary of the $\mathrm{TOL}^{\mathrm{DX}}: 2^{\text {nd }}$ Edition (Culbertson \& Zillmer, 2011) results are provided in Table 1. An example of a Test 1, training, and Test 2 normalized displacement and velocity profile for one participant with ASD is provided in Figure 6. Mean MT, TT, DT, \%TPV, for all participants with ASD are provided in Figure 7. Mean MT, TT, DT, \%TPV, for all the participants who are NT are provided in Figure 8. Mean MT, TT, DT, \%TPV, for all participants are provided in Figure 9.

TABLE 1.1: $\left(\mathrm{TOL}^{\mathrm{DX}}\right)$ : $2^{\text {nd }}$ Edition Participant Classification Scores

\begin{tabular}{|c|c|c|c|c|}
\hline TOL ${ }^{\mathrm{DX}}$ Scores & ASD & NT & TOL $^{\mathrm{DX}}$ Range & $\begin{array}{l}\text { Executive } \\
\text { Planning } \\
\text { Classification }\end{array}$ \\
\hline Total Move & 80.8 & 89.56 & $\geq 130$ & Very Superior \\
\hline Total Correct & 90.8 & 93.11 & $120-129$ & Superior \\
\hline Total Rule Violation & 82.2 & 95.33 & $110-119$ & High Average \\
\hline Total Time Violation* & 83.6 & 102.22 & $90-109$ & Average \\
\hline Total Initiation Time* & 96 & 90.22 & $80-89$ & Low Average \\
\hline Total Execution Time* & 85.8 & 100.89 & $70-79$ & Borderline \\
\hline $\begin{array}{l}\text { Total Problem-Solving } \\
\text { Time* }\end{array}$ & 85.4 & 103.11 & $<70$ & Poor \\
\hline
\end{tabular}

Note. Higher scores indicate positive performance; ASD = Autism Spectrum Disorder Children; $\mathrm{NT}=$ Children who are neurotypical $\mathrm{n}=19 * \mathrm{p}<.10$ 


\subsubsection{Tower of London-Drexel University $\left(\right.$ TOL $\left.^{\mathrm{DX}}\right): 2^{\text {nd }}$ Edition}

The the TOL ${ }^{\mathrm{DX}}: 2^{\text {nd }}$ Edition (Culbertson \& Zillmer, 2011) results indicated no significant difference in Total Move score between the two groups indicating that both groups made a similar number of movements to match the placement of the beads from the examiner's tower ( $p$ $=.362$ ). We also did not find significant differences between the two groups for Total Correct indicating that both groups were able to match theirs beads to those on the examiner's tower using the least amount of movements in concordance to the $\mathrm{TOL}^{\mathrm{DX}}$ score sheet $(\mathrm{p}=.683)$. No significant differences were found between the two groups in Total Rule Violations while completing the task, indicating that both groups of children were able to execute an exercise while being conscious of the rules $(\mathrm{p}=.137)$.

A significant difference was found for Total Time Violation between the two groups indicating that children with ASD took longer than 1 minute to complete an attempt or could not complete the task in 2 minutes. This finding supports Hill (2004), who asserted that it may be extremely challenging and time consuming for individuals with ASD to change a movement plan after it has been created $(\mathrm{p}=.040, \mathrm{M}=-18.622, \mathrm{SD}=8.359)$.

The $\mathrm{TOL}^{\mathrm{DX}}$ manual (Culbertson \& Zillmer, 2011) states that longer initiation times indicate a more structured plan to solve the exercise. The T-test shows no significant differences in Total Initiation Time (how long participants took to start the exercise), indicating that both groups took similar time to constructing their plan. However, this could also be due to children with ASD taking longer to formulate their problem-solving plan, and not due to a betterstructured execution plan. This is supported by the fact that some of the children with ASD could not complete some of the tasks in less than 1 minute $(\mathrm{p}=.062, \mathrm{M}=5.778, \mathrm{SD}=2.896)$. There were no significant differences in Total Execution Time $(\mathrm{p}=.069, \mathrm{M}=-15.089, \mathrm{SD}=7.788)$, 
suggesting that all participants took similar amounts of time to execute an attempt. There was a significant difference in the Total Problem-Solving Time between the two groups. This was due to the children in the ASD group taking longer to execute the exercise $(\mathrm{p}=.037, \mathrm{M}=-17.711$, $\mathrm{SD}=7.811)$.

\subsubsection{Kinematic Data}

The results of the kinematic data are the following. Figure 6 displays a full analysis of a single participant with ASD in the Sine wave condition. The image shows that the child was more than capable of executing the Fitts task in the Test 1 condition. Additionally, although the variable error was high compared to the actual template, the child engaged in fast yet harmonic motion as seen in the training condition. It is important to note that accuracy was never emphasized in the instructions of the Sine wave tracking task. After training with the Sine Wave tracking task, the participants executed the Test 2 Fitts-like task and exhibited faster and more movement attempts in comparison to the Test 1 (see Figure 6). 


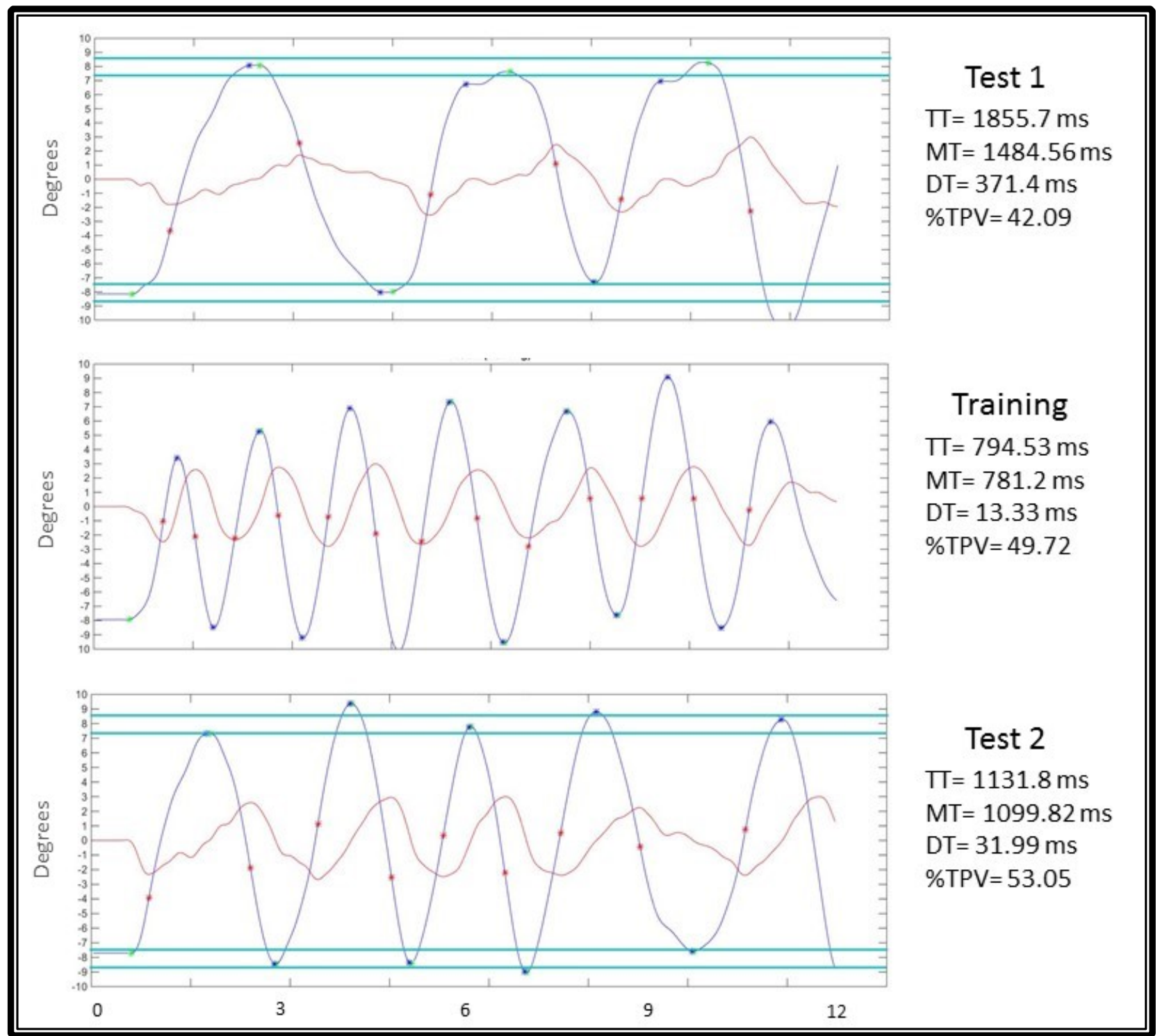

Figure 6: Limb displacement (purple) and velocity trace (red) in Test 1, training, and Test 2 of a single participant with ASD. Red dots on the displacement trace represent points of peak velocity; Blue dots represent movement termination and green represent movement initiation. 


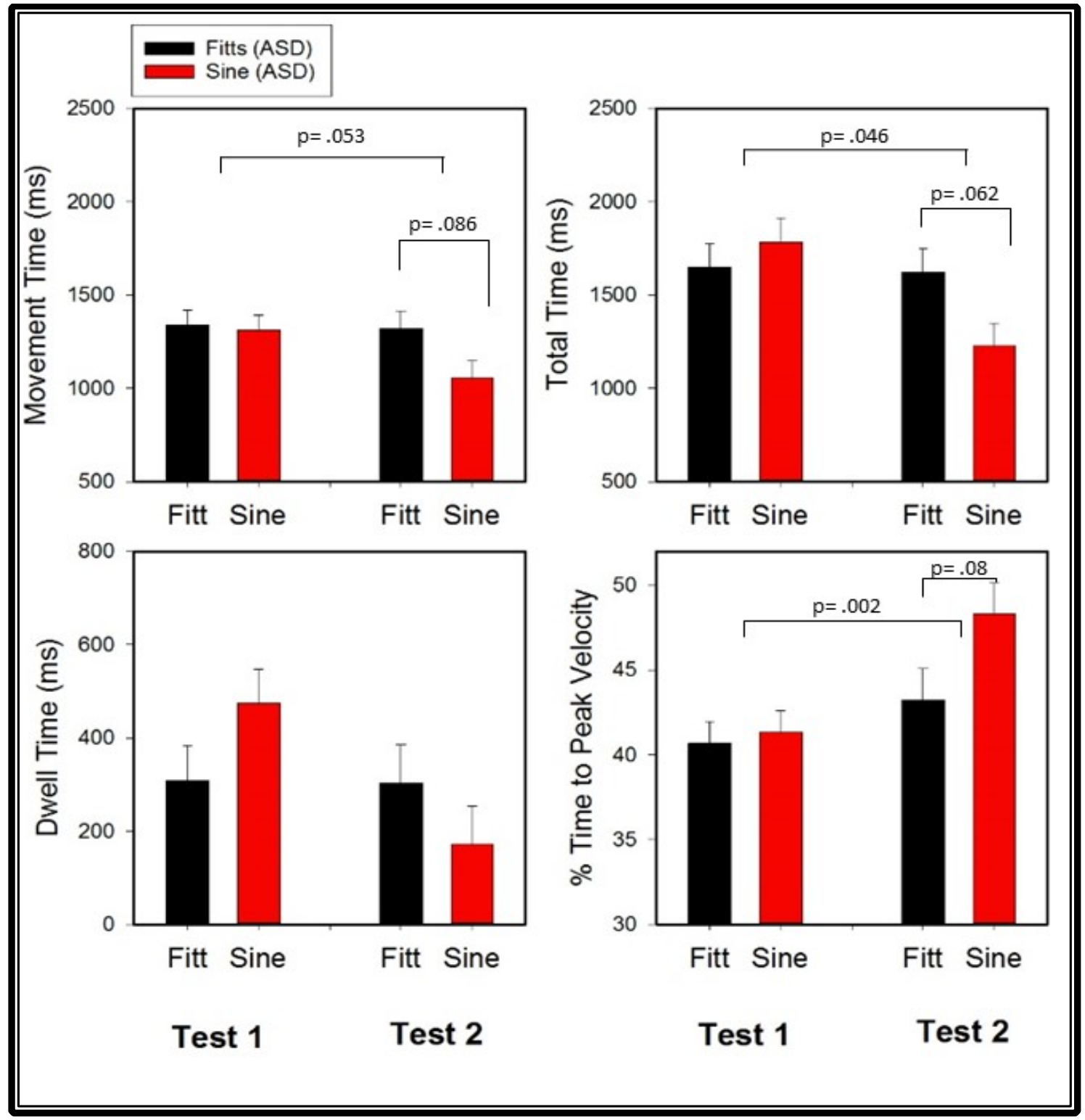

Figure 7: Mean movement time, Total time, Dwell time, and \% time to peak velocity of All Participants with ASD.

\section{Movement Time (MT) of Participants with ASD}

The analysis indicated a within subjects effect for Test, $\mathrm{F}(1,8)=5.164, \mathrm{p}=.053, \eta_{\mathrm{p}}^{2}=.392$, with consistently faster MTs on Test 2 compared to Test1 (Figure 7). Simple main effects analysis for the MT x Condition interaction indicated similar MTs for the Sine Wave and Fitts 
conditions on Test 1 . However, the MTs were significantly shorter for the Sine Wave condition than the Fitts condition on the Test $2, F(1,8)=3.827, p=.086, \eta_{p}^{2}=.324$.

\section{Total Time (TT) of Participants with ASD}

The analysis indicated a within subjects effect for Test, $F(1,8)=5.571, p=.046, \eta_{p}^{2}=$ .410 , with consistently faster TTs on the Test 2 compared to the Test 1 (see Figure 7). Simple main effects analysis for the TT x Condition interaction indicated similar TTs for the Sine Wave and Fitts conditions on the Test 1 . However, the TTs were significantly lower for the Sine Wave condition than the Fitts condition on the Test $2, F(1,8)=4.689, p=.062, \eta_{p}^{2}=.370$.

\section{Dwell Time (DT) of Participants with ASD}

The analysis indicated no significant main effects $(p=.109)$ or interactions $(p=.119)$.

\section{Percent time-to-peak velocity (\% TPV) of Participants with ASD}

The analysis indicated a within subjects effect for Test, $F(1,8)=18.98, p=.002, \eta_{p}^{2}=$ .704, with longer \%TPV on Test 2 compared to Test 1 (Figure 7). Simple main effects analysis for the \%TPV x Condition interaction indicated similar \%TPV for the Sine Wave and Fitts conditions on Test 1 . However, \%TPV was significantly longer for the Sine Wave condition than the Fitts condition on Test $2, F(1,8)=4.006, p=.08, \eta_{p}^{2}=.334$.

To ensure that each participant benefited from tracking the sine wave, individual performance of the children in the Sine Wave condition for MT, DT, and \% TPV are presented in Figure 11. 


\section{Participants who are Neurotypical:}

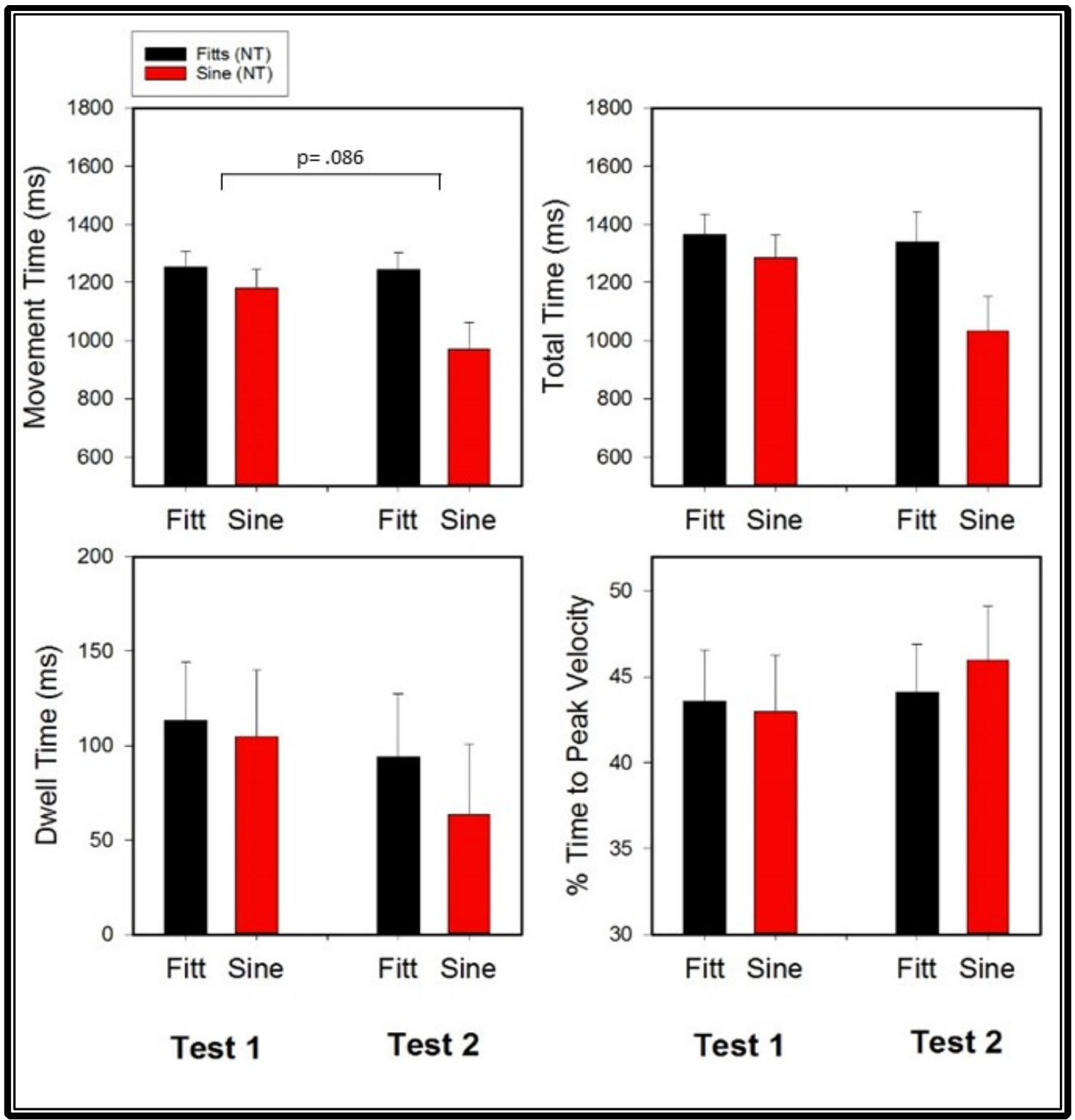

Figure 8: Mean movement time, Total time, Dwell time, and \% time-to-peak velocity of All Neurotypical Participants.

\section{Movement Time (MT) for Participants who are Neurotypical}

The analysis indicated an overall between subjects effect of Condition, $F(1,7)=3.986, \mathrm{p}$

$=.086, \eta_{\mathrm{p}}^{2}=.363$, with consistently lower MT in the Sine condition compared to the Fitts 
condition. The analysis failed to detect any within subjects effect of Test $(\mathrm{p}=.126)$ or Test $\mathrm{x}$ Condition interactions $(\mathrm{p}=.150)$.

Total Time (TT), Dwell Time (DT), and Percent time-to-peak velocity (\% TPV) for

\section{Participants who are Neurotypical}

The analysis failed to detect any between subjects effect of Condition $(p=.121)$, within subjects effect of Test $(p=.126)$, or Test $x$ Condition interactions $(p=.150)$ for TT. The analysis also failed to detect any between subjects effect of Condition $(p=.611)$, within subject effects of Test $(p=.370)$, or Test $x$ Condition interactions $(p=.738)$ for DT; as well as any between subjects effect of Condition $(\mathrm{p}=.883)$, within subject effects of Test $(\mathrm{p}=.20)$, or Test $\mathrm{x}$ Condition interactions $(\mathrm{p}=.348)$ for $\% \mathrm{TPV}$. 


\section{Analysis including all participants:}

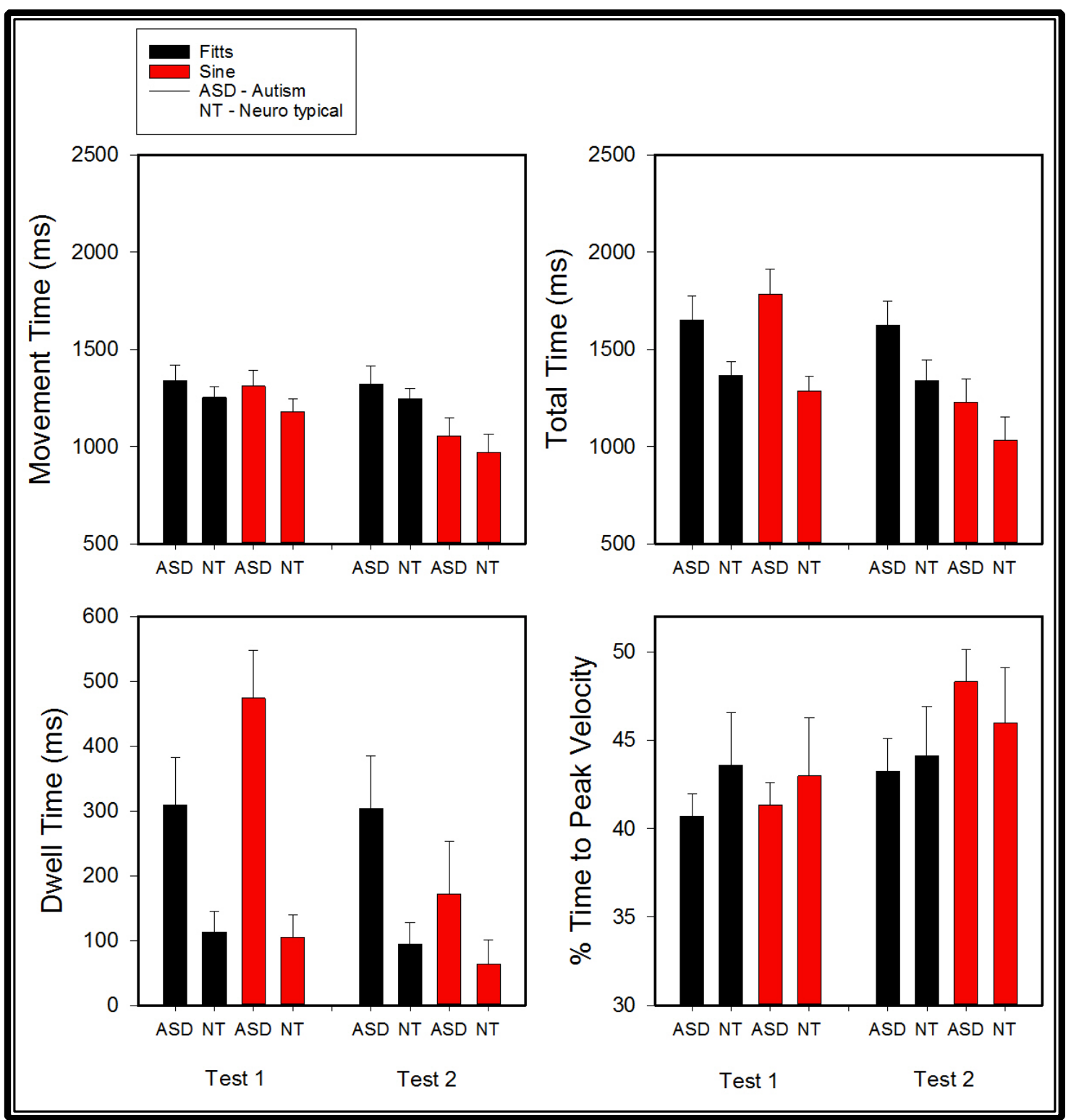

Figure 9: Mean movement time, Total time, Dwell time, and \% time-to-peak velocity of All Participants (ASD group and NT group).

\section{Movement Time (MT)}

The analysis indicated a between subjects effect for Condition, $F(1,15)=5.36, p=.035$, $\eta_{p}^{2}=.263$, with consistently faster MTs in the Sine condition compared to the Fitts condition. The analysis also indicated a within subjects effect for Test, $F(1,15)=7.96, p=.013, \eta 2 p=$ 
.347 , with consistently faster MTs on Test 2 compared to Test 1 , as well as a significant Test $\mathrm{x}$ Condition interaction, $F(1,15)=6.33, p=.024, \eta 2 p=.297$ (see figure 10$)$. The analysis failed to detect any Test x Group interaction $(\mathrm{p}=.759)$ or Test $\mathrm{x}$ Condition $\mathrm{x}$ Group interaction $(\mathrm{p}=.858)$.

\section{Total Time (TT)}

The analysis indicated a between subjects effect for Condition, $F(1,15)=3.73, p=.073$, $\eta_{\mathrm{p}}^{2}=.199$, with consistently faster TTs in the Sine condition compared to Fitts; a between subjects effect for Group, $F(1,15)=14.08, \mathrm{p}=.002, \eta_{\mathrm{p}}^{2}=.484$, with lower TTs in the NT group compared to the ASD group; but failed to detect an interaction of Group x Condition $(\mathrm{p}=.719)$. The analysis indicated a within subjects effect for Test, $\mathrm{F}(1,15)=8.23, \mathrm{p}=.012, \eta 2 \mathrm{p}=.355$, with consistently faster TTs on Test 2 compared to Test 1 , as well as a significant Test $\mathrm{x}$ Condition interaction, $\mathrm{F}(1,15)=6.405, \mathrm{p}=.023, \eta 2 \mathrm{p}=.299$ (see Figure 10). The analysis failed to detect a two-way Test $\mathrm{x}$ Group interaction $(\mathrm{p}=.332)$ or a three-way Test $\mathrm{x}$ Condition $\mathrm{x}$ Group interaction $(\mathrm{p}=.323)$.

\section{Dwell Time (DT)}

The analysis indicated a between subjects effect for Group, $F(1,15)=29.38, p=.000, \eta_{p}^{2}$ $=.662$, with consistently higher DT in the participants with ASD group compared to the NT group. The analysis failed to detect any between subjects effect for Condition $(p=.969)$ as well as the Group $x$ Condition interaction $(\mathrm{p}=.667)$. The analysis indicated a within subjects effect for Test, $F(1,15)=3.72, p=.073, \eta 2 p=.199$, with consistently lower DT on Test 2 compared to Test 1 . The analysis failed to detect significant two-way interactions of Test $x$ Condition $(\mathrm{p}=$ $.115)$ and Test $\mathrm{x}$ Group $(\mathrm{p}=.216)$, or a three-way interaction of Test $\mathrm{x}$ Condition $\mathrm{x}$ Group $(\mathrm{p}=$ $.170)$. 


\section{Percent time-to-peak velocity (\% TPV)}

The analysis failed to detect any between subjects effect for Condition $(\mathrm{p}=.440)$ or Group $(\mathrm{p}=.731)$, or Condition x Group $(\mathrm{p}=.620)$. The analysis indicated a within subjects effect for Test, $\mathrm{F}(1,15)=15.64, \mathrm{p}=.001, \eta 2 \mathrm{p}=.511$, with consistently higher $\%$ TPV on Test 2 compared to Test 1 , as well as a significant Test $\mathrm{x}$ Condition interaction, $\mathrm{F}(1,15)=4.35, \mathrm{p}=$ $.054, \eta 2 p=.225$, with highest values of $\% \mathrm{TPV}$ seen in the Sine Condition on Test 2 . The analysis also found a significant Test $\mathrm{x}$ Group interaction, $\mathrm{F}(1,15)=3.30, \mathrm{p}=.089, \eta 2 \mathrm{p}=.181$, but no three-way Test x Condition x Group interaction $(p=.579)$.

\subsection{DiscuSSION}

The purpose of this study was twofold. First, it was designed to further our understanding of the kinematic composition of goal directed upper limb movement in children diagnosed with ASD by determining where in their movement pattern deficits in motor control were apparent. Furthermore, we wanted to investigate whether and how participants with ASD would adapt their limb movements after practicing a sine wave tracking task. Specifically, we wanted to examine if tracking a sine wave template that provided fast-harmonic movements would aid in the development of faster and smoother velocity profiles in a Fitts-like reciprocal aiming task in participants with ASD. 


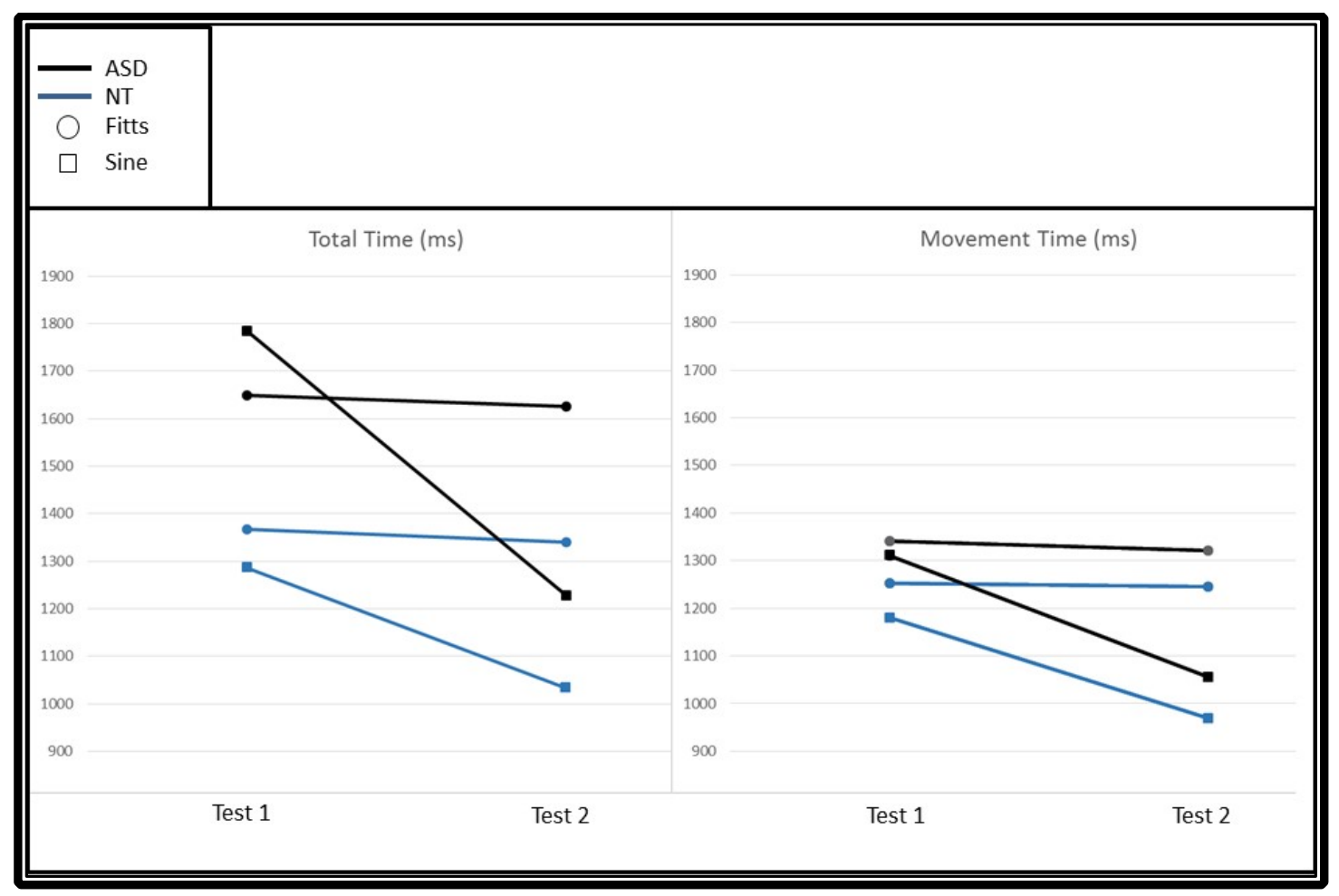

Figure 10: Total time and Mean movement time of All Participants (ASD group and NT group).

The results of the data analysis for the participants with ASD seem to show that they benefited from practicing the Sine wave tracking task, because they were able to reach significantly faster MT and TT in Test 2 for Sine Wave than for Fitts condition (Figure 10). These results indicate that ASD participants were able to use their corrective processes more efficiently to accurately reach the target after practicing goal directed movements in both conditions, but even more so after tracking the sine wave. It is intriguing that although participants with ASD were able to reach faster MTs and TTs, there were no significant differences in their DTs, which means that they took similar time to prepare for the following movement in Test 1 and Test 2 in both the Sine Wave and Fitts conditions. However, similar to MT and TT, \%TPV was higher in Test 2 for both conditions, but \%TPV significantly higher in Test 2 for the Sine Wave condition. These results suggest that in both conditions children with 
ASD were capable of adjusting their motor planning strategy to approach the target more efficiently, but even more so after practicing tracking the sine wave (Figure 10).

These results suggest that children with ASD possibly adopted a new movement structure after practicing goal directed movements in the Sine Wave condition. It appears that children with ASD benefited from tracking the sine wave to improve the ability to make adjustments to their movements on the Fitts-type aiming task (Figure 11). It is important to note that participants were instructed to trace the sine wave to the best of their ability, without mentioning or encouraging speed and accuracy as in the Fitts condition. Participants in the Fitts condition showed smaller performance improvements after practice on the Fitts task supporting the previous work of Boyle et al. (2012a). 

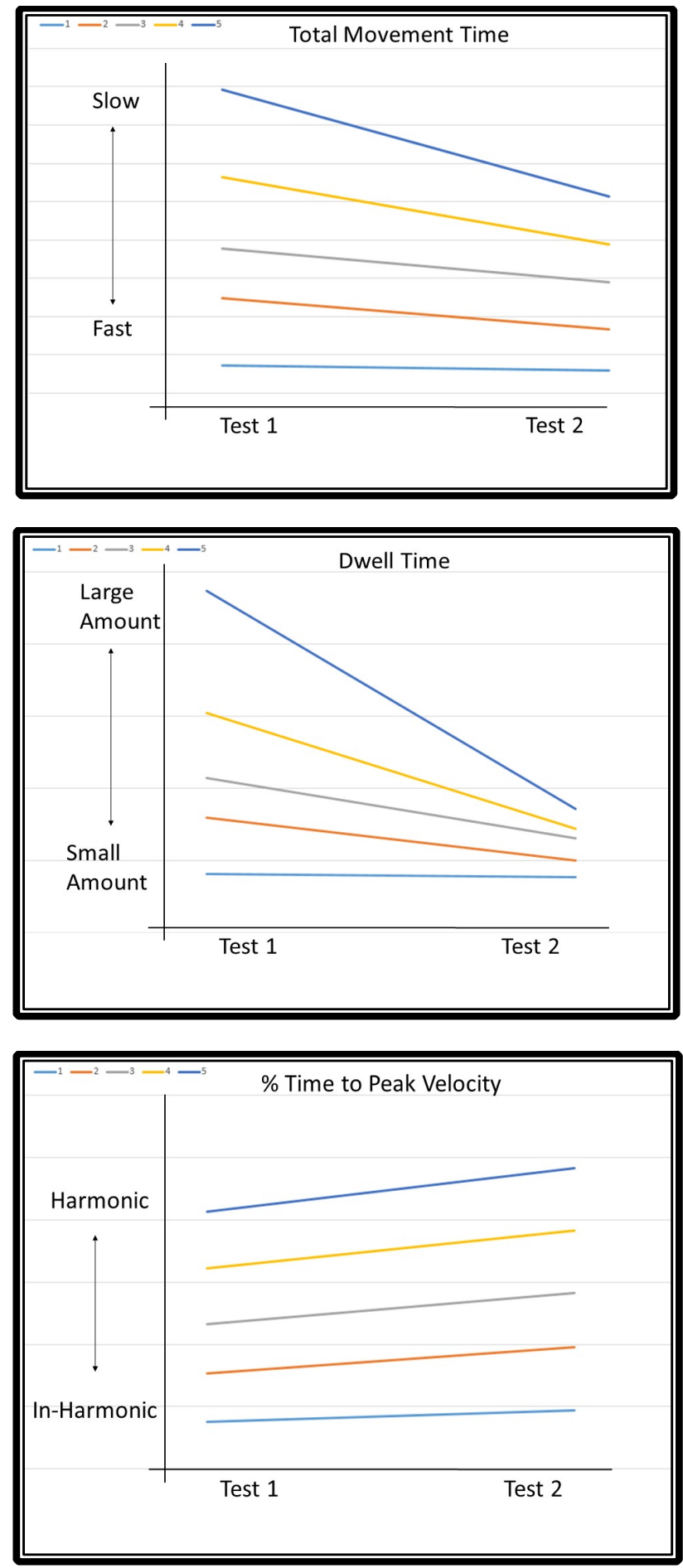

Figure 11: Total movement time, Dwell time, and \% time-to-peak velocity of each participant of the ASD group in the Sine Wave condition. 
The use of visual cues has been found to be effective in helping people with ASD learn and perform multiple tasks (Bryan \& Gast, 2000). In an experiment by Glazebrook et al. (2008), participants had to perform goal directed movements using a concrete motor plan, which was developed by direct and visual cues signaling both the direction and which arm to use in reaching for the required target. When provided with advance visual information about which arm to use and which direction to move in to accurately reach the target, participants with ASD performed similarly compared to the NT group. The findings of the present study agree with the work of Glazebrook et al. (2008) in the sense that people with ASD are capable of using information to enhance their performance.

Moreover, the results of the NT group (Figure 8) indicated no significant differences in TT, DT or \%TPV but a faster overall MT in the Sine Wave condition. This was due to a decrease in time spent pre-planning the movement (not a statistically significantly decrease), an increase in time spent in the primary movement phase (\%TPV), and a faster, more efficient homing-in phase, which suggested a more effective corrective process after practicing the Sine Wave tracking task.

Thomas et al. (2000) reported that the movement substructures in young children as well as in young adults could be enhanced after practicing multiple rapid aiming movements. They examined a group of children of age 6 , age 9 , and a group of young adults age 24 , performing an aiming task similar to the one presented in this experiment but in a discrete fashion. It is important to mention that at baseline, the primary acceleration phase of the movement in young adults consisted of $65 \%-75 \%$ of their total movement time, but only $45 \%$ - $50 \%$ for the 9 years old children, and $25 \%$ - 30\% for the 6 years old children. Thomas et al. (2000) found that after practicing the task, the 9 year olds exhibited the greatest increase (30\%) in the duration of the 
primary acceleration of the movement in comparison to the younger children whose phase increased $24 \%$, and the young adults whose acceleration phase increased $10 \%$. In addition, all the groups were able to improve their overall movement time with the young adults being the fastest and the young children being the slowest. These results refute the notion that maturational factors play a role in enhancing the movement structure of goal directed movements, and show that even young children who are NT are capable of enhancing their movement plans after practice.

In contrast, the NT group in this experiment showed minimal improvements overall in their movement structure after practicing aiming movements in either the Sine Wave or Fitts condition. However, it is important to note that the Sine condition seem to demonstrate a trend towards the positive effects of tracing the sine wave in the overall movement structure of this group. The children were able to significantly decrease their overall movement time in the Sine Wave condition, but the same did not happen over the Fitts condition. It is even more intriguing that after practice the time spent in the acceleration phase of the movement in Test 2 Fitts task increased slightly in both conditions. The findings from the NT group were surprising, because we expected to find results similar to the work by Boyle $(2015,2014,2012 a)$ and Thomas (2000). Further research in to how movement structure of goal directed movements can be adjusted in children is needed to add to the more extensive literature on young adults and the elderly (e.g. Abrams \& Pratt, 1993, Yan, Thomas \& Stelmach, 1998).

Finally, the combined data for the ASD group and the NT group (see Figure 9) indicated that all participants were able to significantly reduce their MT and TT during Test 2 of the Sine Wave condition compared to that of the Fitts condition. Moreover, children in the ASD group of the Sine Wave condition were able to significantly reduce their DT but they still spent more time 
monitoring their movements in comparison to the NT group. Also, both groups were able to significantly improve the time spent in the acceleration phase as shown by higher \%TPV in Test 2 of the Sine Wave condition. This improvement was more pronounced in the ASD group as they had more room to improve than the NT group. These particular findings are important because even though the ASD group remained slower than the NT group, the ASD group in the Sine Wave condition were able to perform the movement in Test 2 with similar TT as the NT group in Test 2 of the Fitts condition.

The results of this particular study somewhat coincide with the findings of three other studies that have analyzed the kinematics of movement structures of people with ASD using the Fitts law speed-accuracy trade-off protocol in their examinations (Glazebrook et al., 2006; Glazebrook et al. 2008; Papadopoulos et al., 2012). No difference in MTs coincides with the work of Papadopoulos et al. (2012), as the authors did not find a difference in the MTs of children with ASD compared to the NT group after performing continuous aiming movements. In contrast, Glazebrook et al. $(2006,2008)$ found that individuals with ASD took longer than the NT group to complete discrete aiming movements. However, higher DTs and taking longer to initiate the movement in children with ASD in our study did coincide with the work of Glazebrook et al. $(2006,2008)$. Although held to accuracy requirements of $90 \%$, our study did not measure the end-point variability. The work of Papadopoulos et al., (2012) found that children with ASD displayed greater end point variability in their movements around the target. Contradicting this finding, Glazebrook et al. (2006) found that individuals with ASD maintained accuracy at the end-point of their movements. An explanation for this divergent result between the studies could be due to Glazebrook et al. (2006) examined young adults on a discrete aiming task while on the other hand Papadopoulos et al. (2012) and the present study, examined children 
on a reciprocal aiming task. It can be inferred from the pattern that is beginning to develop that people with ASD face impairments in their cognitive processes that are dependent on the nature of the two tasks. This is a critical distinction to make given the nature of both discrete and reciprocal movements. In the discrete task, central nervous system's (CNS) noise that could affect planning of the upcoming movement reversal is not present like it would be in the reciprocal aiming task. Allowing a participant to focus on target accuracy without taking into account a subsequent movement has been shown to cause differences in end point kinematics (Buchanan et al., 2006). Further examination of end-point variability of movement trajectories in both reciprocal and discrete movements is necessary to better understand the movement performance deficits children with ASD face.

The limitations of the present study include a wide age range of the participants $(7-12$ years) and a small sample size $(n=19)$; factors that negatively affected statistical power due to the variability in performance that exists within the ASD population. Other limitations of this study are the frequency of the sine wave, which was paced to match the average time seen in previous college-aged individuals' performance studies on a Fitts-type task with an ID $=6$. Also, complications with the software not saving portions of the kinematic data; for this reason the Training condition was excluded from data analysis of all participants as well as all the data collected from the Sine Wave condition of one participant in the NT group.

Future studies should further investigate the visual and proprioceptive sensory inputs involved with tracking the sine wave and attempt to isolate if one input is utilized more than the other. When participants flex and extend the arm bar to match the sine wave, they are using visual information from the sine wave template and their movements as knowledge of performance $(\mathrm{KP})$ and knowledge of results $(\mathrm{KR})$. In order to isolate the importance of 
concurrent visual feedback or feed-forward, future experiments should isolate the visual input to simply seeing the task traced without the displaying the real-time trace representing the physical action of moving the arm bar. A recent study by Boyle and colleagues (personal communication) examined college-aged participants visually observing the sine wave being traced and its role in upper extremity control. Following 45 trials of viewing the sine wave being tracked, analysis of movement kinematics showed no change in motor performance, leading the authors to conclude that in order for the sine wave to enhance motor performance, the participant needed to actually perform the movement and obtain the proprioceptive feedback of their movement in order to reconstruct the goal directed movement plan. Interestingly though, the participants observing the sine wave did show a significant increase in \%TPV, leading the authors to suggest that the act of visually observing the sine wave may increase the harmonic nature of the movement without the physically experiencing the task, but not improve some features of the movement. Future studies of motor control in individuals with ASD should consider examining their performance in similar conditions

Other interesting topics left unanswered are the lack of differences in TT, DT, and \%TPV, after undergoing practice in the Sine Wave and Fitts conditions in the NT group. As previous work by Boyle et al. $(2015,2014,2012 a)$ and Thomas et al. (2000) have demonstrated differences in these variables within children, young adults, and the elderly. Also the difference of movement trajectories in discrete and reciprocal movements in children with ASD should continue to be examined. Lastly, future studies should investigate if the benefits obtained by practicing the sine wave tracking task are transferrable to discrete aiming or grasping movement tasks such as reaching for a glass of water. 


\subsection{CONCLUSION}

Children with ASD spent more time planning and correcting their movements than children who are NT, suggesting impairments in both motor planning and motor control processes. However, physical practice of a sine wave movement using an optimized template that provided fast-harmonic motion, seem effectively to aid in the development of faster and smoother velocity profiles in the homing-in phase of participants with ASD.

Furthermore, when comparing both the ASD group and the NT group under the Sine Wave and Fitts condition, they were able to perform overall faster and more harmonic movements by improving all the movement substructures of a goal directed movement. Both groups were able to decrease the time spent in pre-planning their movements, improve the time spent in the primary movement phase, thus getting closer to the target, and decrease the time spent correcting their original motor plan to accurately reach the target. Note that all movement structure improvements were significantly notable under the Sine Wave condition. Supporting the notion that children with ASD as well as children who are NT may benefit from practice to improve their overall movement structure due to both the Sine Wave and Fitts conditions resulted in improvements in the overall movement structure of the two groups.

The results of the Sine Wave condition of the participants with ASD in this experiment suggested that they benefited from tracking the sine wave to learn how to execute smoother reciprocal goal directed movements by excluding the concern of hitting the targets, which is constantly present in the Fitts condition. As a result, when the sine wave template was removed, the children with ASD seem to have retained the movement control characteristics previously learned by following the sine wave. In other words, the children with ASD who were exposed to the sine wave, showed a capacity to adopt and modify their movement structure to perform in a 
similar manner as children who are NT in a slightly different goal directed task. Furthermore, practice in the Fitts condition resulted in less movement improvement on Test 2 than the Sine Wave condition. It is important to remark that based on the theory of specificity, this condition was expected to have resulted in the best performance on Test 2. 


\section{References}

Abrams, R. A., \& Pratt, J. (1993). Rapid aimed limb movements: Differential effects of practice on component submovements. Journal of motor behavior, 25(4), 288-298.

Adam, J. J., \& Paas, F. G. (1996). Dwell time in reciprocal aiming tasks. Human Movement Science, 15(1), 1-24.

Adrien, J. L., Lenoir, P., Martineau, J., Perrot, A., Hameury, L., Larmande, C., \& Sauvage, D. (1993). Blind ratings of early symptoms of autism based upon family home movies. Journal of the American Academy of Child \& Adolescent Psychiatry, 32(3), 617-626.

Allen, G. (2005). The cerebellum in autism. Clin Neuropsychiatry, 2(6), 321-337.

American Psychiatric Association. (2013). Diagnostic and statistical manual of mental disorders (DSM-5®). American Psychiatric Pub.

American Psychiatric Association, \& American Psychiatric Association. (1994). Diagnostic and statistical manual of mental disorders (DSM). Washington, DC: American psychiatric association, 143-7.

Asperger, H. (1944). Die „Autistischen Psychopathen” im Kindesalter. European Archives of Psychiatry and Clinical Neuroscience, 117(1), 76-136.

Asperger, H., \& Frith, U. T. (1991). Autistic psychopathy in childhood.

Bailey, A., Le Couteur, A., Gottesman, I., Bolton, P., Simonoff, E., Yuzda, E., \& Rutter, M. (1995). Autism as a strongly genetic disorder: evidence from a British twin study. Psychological medicine, 25(01), 63-77.

Baranek, G. T. (1999). Autism during infancy: A retrospective video analysis of sensory-motor and social behaviors at 9-12 months of age. Journal of autism and developmental disorders, 29(3), 213-224. 
Bauman, M. L. (1992). Motor dysfunction in autism. Movement disorders in neurology and psychiatry, 1, 658-661.

Beggs, W. D. A., \& Howarth, C. I. (1970). Movement control in a repetitive motor task. Nature, 225(5234), 752-753.

Bhat, A. N., Galloway, J. C., \& Landa, R. J. (2012). Relation between early motor delay and later communication delay in infants at risk for autism. Infant Behavior and Development, 35(4), 838-846.

Bolton, P., Macdonald, H., Pickles, A., Rios, P. A., Goode, S., Crowson, M. \& Rutter, M. (1994). A case-control family history study of autism. Journal of Child Psychology and Psychiatry, 35(5), 877-900.

Boyle, J. B., \& Shea, C. H. (2011). Wrist and arm movements of varying difficulties. Acta psychologica, 137(3), 382-396.

Boyle, J., Panzer, S., Wright, D., \& Shea, C. H. (2012a). Extended practice of reciprocal wrist and arm movements of varying difficulties. Acta psychologica, 140(2), 142-153.

Boyle, J.B., Kennedy, D., \& Shea, C.H. (2012b). Optimizing the control of high ID single degree of freedom movements: Re-thinking the obvious. Experimental Brain Research, 223, 377-387.

Boyle, J.B., Kennedy, D., Wang, C., \& Shea, C.H. (2014). The sine wave protocol: Decreasing movement time without increasing errors. Journal of Motor Behavior, 46, 233-241.

Boyle, J.B., Kennedy, D.M., \& Shea, C.H. (2015). A novel approach to enhancing limb control in older adults. Experimental Brain Research, 233, 2061-2071.

Boyle, J.B., Kennedy, D., Wang, C., \& Shea, C.H. (2016). Optimizing high ID performance: The role of the tracking template. Journal of Motor Learning and Development, 4.1, 80-99.

Brambilla, P., Hardan, A., di Nemi, S. U., Perez, J., Soares, J. C., \& Barale, F. (2003). Brain anatomy and development in autism: review of structural MRI studies. Brain research bulletin, 61(6), 557-569.

Bryan, L. C., \& Gast, D. L. (2000). Teaching on-task and on-schedule behaviors to high- 
functioning children with autism via picture activity schedules. Journal of autism and developmental disorders, 30(6), 553-567.

Buchanan, J. J., Park, J. H., \& Shea, C. H. (2006). Target width scaling in a repetitive aiming task: switching between cyclical and discrete units of action. Experimental Brain Research, 175(4), 710-725.

Burton, A. W., \& Miller, D. E. (1998). Movement skill assessment. Human Kinetics.

Campione, G. C., Piazza, C., Villa, L., \& Molteni, M. (2016). Three-dimensional kinematic analysis of prehension movements in young children with autism spectrum disorder: new insights on motor impairment. Journal of autism and developmental disorders, 46(6), 1985-1999.

Carlton, L. G. (1981). Visual information: The control of aiming movements. The Quarterly Journal of Experimental Psychology, 33(1), 87-93.

Cattaneo, L., Fabbri-Destro, M., Boria, S., Pieraccini, C., Monti, A., Cossu, G., \& Rizzolatti, G. (2007). Impairment of actions chains in autism and its possible role in intention understanding. Proceedings of the National Academy of Sciences, 104(45), 17825-17830.

Christensen, D. L., Bilder, D. A., Zahorodny, W., Pettygrove, S., Durkin, M. S., Fitzgerald, R. T. \& Yeargin-Allsopp, M. (2016). Prevalence and characteristics of autism spectrum disorder among 4-year-old children in the autism and developmental disabilities monitoring network. Journal of Developmental \& Behavioral Pediatrics, 37(1), 1-8.

Coull, J., Tremblay, L., \& Elliott, D. (2001). Examining the specificity of practice hypothesis: is learning modality specific? Research quarterly for exercise and sport, 72(4), 345-354.

Culbertson, W. C., Zillmer, E. A. (2011). Tower of London - Drexel University 2nd Edition Technical Manual. North Tonawanda, NY: Multi-Health Systems Inc.

David, F. J., Baranek, G. T., Giuliani, C. A., Mercer, V. S., Poe, M. D., \& Thorpe, D. E. (2009). A pilot study: coordination of precision grip in children and adolescents with high functioning autism. Pediatric Physical Therapy, 21(2), 205.

David, F. J., Baranek, G. T., Wiesen, C., Miao, A., \& Thorpe, D. E. (2012). Coordination of 
precision grip in 2-6 years-old children with autism spectrum disorders compared to children developing typically and children with developmental disabilities. Frontiers in integrative neuroscience, 6,122 .

Debaere, F., Wenderoth, N., Sunaert, S., Van Hecke, P., \& Swinnen, S. P. (2003). Internal vs external generation of movements: differential neural pathways involved in bimanual coordination performed in the presence or absence of augmented visual feedback. Neuroimage, 19(3), 764-776.

Diagnosis, Causes \& Symptoms. (2016). Autism Speaks Inc. Autism Speaks and Autism Speaks It's Time To Listen \& Design are registered trademarks owned by Autism Speaks Inc. Retrieved October 15, 2016, from https://www.autismspeaks.org/family-services/toolkits/100-day-kit/diagnosis-causes-symptoms

Dowd, A. M., McGinley, J. L., Taffe, J. R., \& Rinehart, N. J. (2012). Do planning and visual integration difficulties underpin motor dysfunction in autism? A kinematic study of young children with autism. Journal of autism and developmental disorders, 42(8), 15391548.

Elliott, D. (2010). Vision and goal-directed movement: neurobehavioral perspectives. Human Kinetics.

Elliott, D., Hansen, S., Mendoza, J., \& Tremblay, L. (2004). Learning to optimize speed, accuracy, and energy expenditure: a framework for understanding speed-accuracy relations in goal-directed aiming. Journal of motor behavior, 36(3), 339-351.

Fabbri-Destro, M., Cattaneo, L., Boria, S., \& Rizzolatti, G. (2009). Planning actions in autism. Experimental Brain Research, 192(3), 521-525.

Fitts, P.M. (1954) The information capacity of the human motor system in controlling the amplitude of movement. Journal of Experimental Psychology 47,381-391.

Fitts, P. M., \& Peterson, J. R. (1964). Information capacity of discrete motor responses. Journal of experimental psychology, 67(2), 103.

Forti, S., Valli, A., Perego, P., Nobile, M., Crippa, A., \& Molteni, M. (2011). Motor planning 
and control in autism. A kinematic analysis of preschool children. Research in Autism Spectrum Disorders, 5(2), 834-842.

Fournier, K. A., Hass, C. J., Naik, S. K., Lodha, N., \& Cauraugh, J. H. (2010). Motor coordination in autism spectrum disorders: a synthesis and meta-analysis. Journal of autism and developmental disorders, 40(10), 1227-1240.

Freitag, C. M., Kleser, C., Schneider, M., \& von Gontard, A. (2007). Quantitative assessment of neuromotor function in adolescents with high functioning autism and Asperger syndrome. Journal of autism and developmental disorders, 37(5), 948-959.

Glazebrook, C. M., Elliott, D., \& Lyons, J. (2006). A kinematic analysis of how young adults with and without autism plan and control goal-directed movements. Motor control, 10(3), 244-264.

Glazebrook, C. M., Elliott, D., \& Szatmari, P. (2008). How do individuals with autism plan their movements? Journal of Autism and Developmental Disorders, 38(1), 114-126.

Green, D., Charman, T., Pickles, A., Chandler, S., Loucas, T., Simonoff, E., et al. (2009). Impairment in movement skills of children with autistic spectrum disorder. Developmental Medicine and Child Neurology, 51, 311-316.

Guiard, Y. (1997). Fitts' law in the discrete vs. cyclical paradigm. Human Movement Science, 16(1), 97-131.

Hughes, C. (1996). Brief report: Planning problems in autism at the level of motor control. Journal of autism and developmental disorders, 26(1), 99-107.

H. Yan Jerry R. Thomas George E. Stelmach, J. (1998). Aging and rapid aiming arm movement control. Experimental aging research, 24(2), 155-168.

Isaksen, J., Bryn, V., Diseth, T. H., Heiberg, A., Schjølberg, S., \& Skjeldal, O. H. (2013). Children with autism spectrum disorders-The importance of medical investigations. European Journal of Paediatric Neurology, 17(1), 68-76.

Itzchak, E. B., Lahat, E., \& Zachor, D. A. (2011). Advanced parental ages and low birth weight 
in autism spectrum disorders-Rates and effect on functioning. Research in developmental disabilities, 32(5), 1776-1781.

Kanner, L. (1943). Autistic disturbances of affective contact. Nervous child, 2(3), 217-250.

Kelso, B. A. (1995). The effects of extended practice on aiming movements in terms of Fitts' Law. York University.

Ketcham, C. J., Seidler, R. D., Van Gemmert, A. W., \& Stelmach, G. E. (2002). Age-related kinematic differences as influenced by task difficulty, target size, and movement amplitude. The Journals of Gerontology Series B: Psychological Sciences and Social Sciences, 57(1), P54-P64.

Kohen-Raz, R., Volkman, F. R., \& Cohen, D. J. (1992). Postural control in children with autism. Journal of autism and developmental disorders, 22(3), 419-432.

Kovacs, A. J., Buchanan, J. J., \& Shea, C. H. (2008). Perceptual influences on Fitts' law. Experimental Brain Research, 190(1), 99-103.

Kovacs, A. J., \& Shea, C. H. (2011). The learning of 90 continuous relative phase with and without Lissajous feedback: external and internally generated bimanual coordination. Acta psychologica, 136(3), 311-320.

Landa, R., \& Garrett-Mayer, E. (2006). Development in infants with autism spectrum disorders: a prospective study. Journal of Child Psychology and Psychiatry, 47(6), 629638.

Landrigan, P. J. (2010). What causes autism? Exploring the environmental contribution. Current opinion in pediatrics, 22(2), 219-225.

Leary, M. R., \& Hill, D. A. (1996). Moving on: autism and movement disturbance. Mental retardation, 34(1), 39 .

Leigh, J. P., \& Du, J. (2015). Brief report: forecasting the economic burden of autism in 2015 and 2025 in the United States. Journal of autism and developmental disorders, 45(12), 4135-4139.

Loh, A., Soman, T., Brian, J., Bryson, S. E., Roberts, W., Szatmari, P., ... \& Zwaigenbaum, L. 
(2007). Stereotyped motor behaviors associated with autism in high-risk infants: A pilot videotape analysis of a sibling sample. Journal of autism and developmental disorders, $37(1), 25-36$.

Magill, R. A., \& Anderson, D. I. (2007). Motor learning and control: Concepts and applications (Vol. 11). New York: McGraw-Hill.

Mari, M., Castiello, U., Marks, D., Marraffa, C., \& Prior, M. (2003). The reach-to-grasp movement in children with autism spectrum disorder. Philosophical Transactions of the Royal Society of London B: Biological Sciences, 358(1430), 393-403.

Meyer, D. E., Abrams, R. A., Kornblum, S., Wright, C. E., \& Keith Smith, J. E. (1988). Optimality in human motor performance: ideal control of rapid aimed movements. Psychological review, 95(3), 340.

Ming, X., Brimacombe, M., \& Wagner, G. C. (2007). Prevalence of motor impairment in autism spectrum disorders. Brain and Development, 29(9), 565-570.

Minshew, N. J., Sung, K., Jones, B. L., \& Furman, J. M. (2004). Underdevelopment of the postural control system in autism. Neurology, 63(11), 2056-2061.

Molloy, C. A., Dietrich, K. N., \& Bhattacharya, A. (2003). Postural stability in children with autism spectrum disorder. Journal of autism and developmental disorders, 33(6), 643652.

Mosconi, M. W., Mohanty, S., Greene, R. K., Cook, E. H., Vaillancourt, D. E., \& Sweeney, J. A. (2015). Feedforward and feedback motor control abnormalities implicate cerebellar dysfunctions in autism spectrum disorder. Journal of Neuroscience, 35(5).

Mottet, D., \& Bootsma, R. J. (1999). The dynamics of goal-directed rhythmical aiming. Biological cybernetics, 80(4), 235-245.

Mugnaini, E. (1972). The histology and cytology of the cerebellar cortex. The Comparative Anatomy and Histology of the Cerebellum, 201-262.

Nazarali, N., Glazebrook, C. M., \& Elliott, D. (2009). Movement planning and reprogramming in individuals with autism. Journal of Autism and Developmental Disorders, 39(10), 
$1401-1411$

Ozonoff, S., Young, G. S., Goldring, S., Greiss-Hess, L., Herrera, A. M., Steele, J., ... \& Rogers, S. J. (2008). Gross motor development, movement abnormalities, and early identification of autism. Journal of autism and developmental disorders, 38(4), 644-656.

Palmen, S. J., van Engeland, H., Hof, P. R., \& Schmitz, C. (2004). Neuropathological findings in autism. Brain, 127(12), 2572-2583.

Papadopoulos, N., McGinley, J., Tonge, B. J., Bradshaw, J. L., Saunders, K., \& Rinehart, N. J. (2012). An investigation of upper limb motor function in high functioning autism and Asperger's disorder using a repetitive Fitts' aiming task. Research in Autism Spectrum Disorders, 6(1), 286-292.

Proteau, L. (1995). Sensory integration in the learning of an aiming task. Canadian Journal of Experimental Psychology/Revue canadienne de psychologie expérimentale, 49(1), 113.

Provost, B., Lopez, B. R., \& Heimerl, S. (2007). A comparison of motor delays in young children: autism spectrum disorder, developmental delay, and developmental concerns. Journal of autism and developmental disorders, 37(2), 321-328.

Rinehart, N. J., Bellgrove, M. A., Tonge, B. J., Brereton, A. V., Howells-Rankin, D., \& Bradshaw, J. L. (2006). An examination of movement kinematics in young people with high-functioning autism and Asperger's disorder: further evidence for a motor planning deficit. Journal of autism and developmental disorders, 36(6), 757-767.

Rogers, S. J., Hepburn, S. L., Stackhouse, T., \& Wehner, E. (2003). Imitation performance in toddlers with autism and those with other developmental disorders. Journal of child psychology and psychiatry, 44(5), 763-781.

Rosenbaum, D. A. (1991) Human motor control. London: Academic Press Limited.

Sacrey, L. A. R., Armstrong, V. L., Bryson, S. E., \& Zwaigenbaum, L. (2014). Impairments to visual disengagement in autism spectrum disorder: a review of experimental studies from infancy to adulthood. Neuroscience \& Biobehavioral Reviews, 47, 559-577.

Schaafsma, S. M., \& Pfaff, D. W. (2014). Etiologies underlying sex differences in autism 
spectrum disorders. Frontiers in neuroendocrinology, 35(3), 255-271.

Schmidt, R. A., \& Wulf, G. (1997). Continuous concurrent feedback degrades skill learning: Implications for training and simulation. Human Factors: The Journal of the Human Factors and Ergonomics Society, 39(4), 509-525.

Schmitz, C., Martineau, J., Barthélémy, C., \& Assaiante, C. (2003). Motor control and children with autism: deficit of anticipatory function?. Neuroscience letters, 348(1), 17-20.

Shannon, C. E. (1949). Communication theory of secrecy systems. Bell Labs Technical Journal, 28(4), 656-715.

Sivaswamy, L., Kumar, A., Rajan, D., Behen, M., Muzik, O., Chugani, D., \& Chugani, H. (2010). A diffusion tensor imaging study of the cerebellar pathways in children with autism spectrum disorder. Journal of child neurology.

Staples, K. L., \& Reid, G. (2010). Fundamental movement skills and autism spectrum disorders. Journal of autism and developmental disorders, 40(2), 209-217.

Sukhodolsky, D. G., Scahill, L., Gadow, K. D., Arnold, L. E., Aman, M. G., McDougle, C. J., ... \& Vitiello, B. (2008). Parent-rated anxiety symptoms in children with pervasive developmental disorders: Frequency and association with core autism symptoms and cognitive functioning. Journal of abnormal child psychology, 36(1), 117-128.

Teitelbaum, P., Teitelbaum, O., Nye, J., Fryman, J., \& Maurer, R. G. (1998). Movement analysis in infancy may be useful for early diagnosis of autism. Proceedings of the National Academy of Sciences, 95(23), 13982-13987.

Teitelbaum, O., Benton, T., Shah, P. K., Prince, A., Kelly, J. L., \& Teitelbaum, P. (2004). Eshkol-Wachman movement notation in diagnosis: The early detection of Asperger's syndrome. Proceedings of the National Academy of Sciences of the United States of America, 101(32), 11909-11914.

Thomas, J. R., Yan, J. H., \& Stelmach, G. E. (2000). Movement substructures change as a function of practice in children and adults. Journal of experimental child psychology, 75(3), 228-244. 
Van Vuchelen, M., Roeyers, H., \& De Weerdt, W. (2007). Nature of motor imitation problems in school-aged males with autism: how congruent are the error types? Developmental Medicine \& Child Neurology, 49(1), 6-12.

Vilensky, J. A., Damasio, A. R., \& Maurer, R. G. (1981). Gait disturbances in patients with autistic behavior: a preliminary study. Archives of neurology, 38(10), 646-649.

Walker, N., Philbin, D., Worden, A., \& Smelcer, J. B. (1997). A program for parsing mouse movements into component submovements. Behavior Research Methods, Instruments, \& Computers, 29(3), 456-460.

Whitehouse, A. (2016). What causes autism? What we know, don't know and suspect. The Conversation US, Inc. Retrieved from http://theconversation.com/what-causes-autismwhat-we-know-dont-know-and-suspect-53977

Wing, L. (1981). Asperger's syndrome: a clinical account. Psychological medicine, 11(1), 115129.

Wing, L. (1997). The History of Ideas on Autism Legends, Myths and Reality. Autism, 1(1), 1323.

Wing, L., \& Potter, D. (2002). The epidemiology of autistic spectrum disorders: is the prevalence rising?. Mental retardation and developmental disabilities research reviews, 8(3), 151161.

Woodworth, R. S. (1899). Accuracy of voluntary movement. The Psychological Review: Monograph Supplements, 3(3), i. 


\section{Vita}

Ms. Alejandra Gamez obtained both B.S. and M.S. in Kinesiology from The University of Texas at El Paso. Ms. Gamez worked as a research assistant where she was exposed to study different areas within the field of biomechanics and motor control. She has published two abstracts in the Journal of Sport and Exercise Psychology, "A novel approach to enhancing upper extremity coordination in children with autism spectrum disorder" and "Single and dual leg Fitts task: Is two better than one?" Additionally Ms. Gamez co-authored a manuscript titled "Preflight adjustments: The role of Fitts law in a two footed forward leap", which is in revision by Human Movement Science.

Ms. Gamez received an award from the Organization for Autism Research to investigate coordination issues in children diagnosed with autism spectrum disorder. In addition to this award, Ms. Gamez received an invitation to present her project at the North American Society for the Psychology of Sport and Physical Activity annual conference and also at OCALICON, the Nation's Premier Autism and Disabilities Conference.

Aside from research, Ms. Gamez worked over two years as a Teaching Assistant for the department of kinesiology and was in charge of teaching the laboratory portion of the biomechanics course for undergraduate students.

Permanent address: Carretera Panamericana Km. 951 Colonia Santa Fe

Durango, Durango, Mexico 34240

This thesis was typed by Alejandra S. Gamez Corral. 